\title{
Search for Long-Lived Particles Decaying in the CMS End Cap Muon Detectors in Proton-Proton Collisions at $\sqrt{s}=13 \mathrm{TeV}$
}

\author{
A. Tumasyan et al. \\ (CMS Collaboration)
}

(Received 10 July 2021; revised 18 September 2021; accepted 26 October 2021; published 23 December 2021)

\begin{abstract}
A search for long-lived particles (LLPs) produced in decays of standard model (SM) Higgs bosons is presented. The data sample consists of $137 \mathrm{fb}^{-1}$ of proton-proton collisions at $\sqrt{s}=13 \mathrm{TeV}$, recorded at the LHC in 2016-2018. A novel technique is employed to reconstruct decays of LLPs in the end cap muon detectors. The search is sensitive to a broad range of LLP decay modes and to masses as low as a few GeV. No excess of events above the SM background is observed. The most stringent limits to date on the branching fraction of the Higgs boson to LLPs subsequently decaying to quarks and $\tau^{+} \tau^{-}$are found for proper decay lengths greater than 6, 20, and $40 \mathrm{~m}$, for LLP masses of 7, 15, and $40 \mathrm{GeV}$, respectively.
\end{abstract}

DOI: 10.1103/PhysRevLett.127.261804

Many extensions of the standard model (SM) predict the existence of neutral, weakly coupled particles that have a long lifetime. These long-lived particles (LLPs) naturally arise in models of split supersymmetry (SUSY) [1-6], SUSY with weak $R$-parity violation [7-10], SUSY with gauge-mediated supersymmetry breaking [11-13], stealth SUSY [14,15], hidden valley scenarios [16-18], baryogenesis triggered by weakly interacting massive particles [19-21], inelastic dark matter [22], and twin Higgs models [23-25].

In this Letter, we describe the first search at the LHC that uses a muon detector as a sampling calorimeter to identify showers produced by decays of LLPs. The CMS end cap muon detectors (EMD) are composed of detector planes interleaved with the steel layers of the magnet flux-return yoke. A schematic diagram of the EMD geometry is shown in Fig. 1. Decays of LLPs in the EMD induce hadronic and electromagnetic showers, giving rise to a high hit multiplicity in localized detector regions. The hadron calorimeter, solenoid magnet, and steel flux-return yoke together provide 20-27 nuclear interaction lengths of shielding, which is sufficiently large to suppress particle showers that are not fully contained (punch through) to negligible levels.

This search has sensitivity to singly or multiply produced LLPs decaying to final states including hadrons, taus, electrons, or photons. The LLPs decaying to muons will very rarely produce a particle shower and will remain undetected by this search. We focus on a benchmark

*Full author list given at the end of the article.

Published by the American Physical Society under the terms of the Creative Commons Attribution 4.0 International license. Further distribution of this work must maintain attribution to the author(s) and the published article's title, journal citation, and DOI. Funded by SCOAP ${ }^{3}$. simplified model motivated by the twin Higgs scenario [16-18,26-28] where the SM Higgs boson $(H)$ decays to a pair of neutral long-lived scalars $(S)$, each of which decays in turn to a pair of bottom quarks $(b \bar{b}), \tau$ leptons $\left(\tau^{+} \tau^{-}\right)$, or down quarks $(d \bar{d})$. Further details of the simplified model can be found in Ref. [29]. The most stringent previous limit for mean proper decay lengths $c \tau<0.3 \mathrm{~m}$ is based on a search for displaced jets in the CMS tracker [29]. For $c \tau>0.3 \mathrm{~m}$, displaced vertices in the ATLAS muon spectrometer [30,31] set the most stringent previous limit.

There are two inherent advantages of using the CMS EMD as a sampling calorimeter to detect LLPs over past

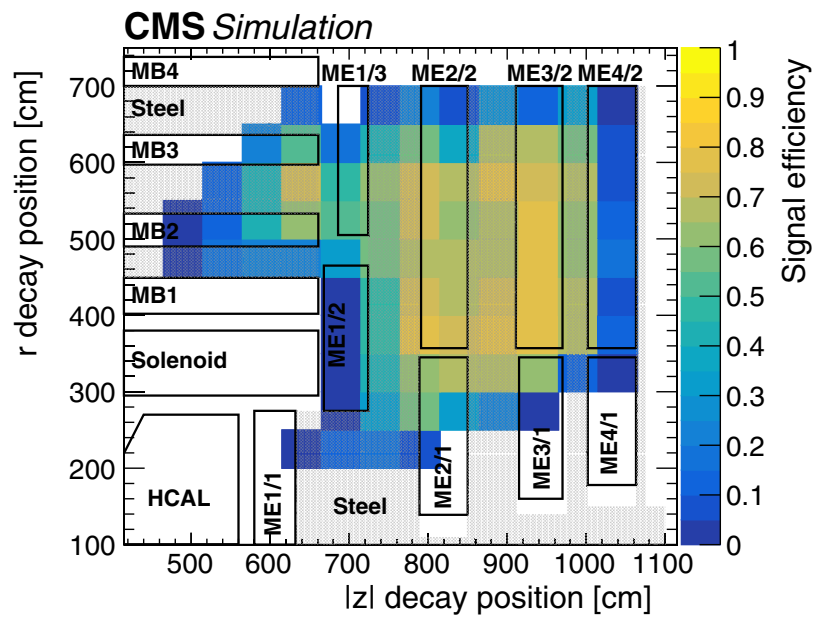

FIG. 1. The signal efficiency of the combined cluster reconstruction, veto, and identification selections as a function of the simulated $r$ and $z$ decay positions of $S$ decaying to $b \bar{b}$, for a mass of $15 \mathrm{GeV}$ and a uniformly distributed mixture of events with $c \tau$ between 1-10 $\mathrm{m}$. The barrel and end cap muon stations are drawn as black boxes and labeled by their station names, showing the geometry of the muon detectors. Regions occupied by the steel return yoke are shaded in gray. 
searches that employ displaced vertices: (i) the absorber material in front of the EMD acts as a shield to maintain a sufficiently low level of background with the detection of a single LLP decay, a level that current displaced vertex searches can only achieve by requiring the detection of two LLP decays; (ii) the calorimetric nature of the particle shower, sensitive to the LLP energy rather than its mass, renders this search equally sensitive to all LLP masses considered, while vertex reconstruction efficiency tends to decrease with the LLP mass due to increasingly smaller opening angles.

Because of these advantages, the signal acceptance and sensitivity are improved relative to the previous best results $[30,31]$ by more than a factor of 6 (2) for an LLP mass of $7 \mathrm{GeV}(\geq 15) \mathrm{GeV}$ and $c \tau>100 \mathrm{~m}$. The improved signal acceptance that results from not requiring a second detected LLP decay is particularly powerful at very large $c \tau$, making the present search the first to be sensitive to LLP decays with $c \tau$ up to $1000 \mathrm{~m}$ and mass between 40 and $55 \mathrm{GeV}$ for the Higgs boson to LLP decay branching fractions below $20 \%$. Tabulated results and instructions to reproduce the signal efficiency are provided in HEPData [32].

The search is based on proton-proton $(p p)$ collision data at $13 \mathrm{TeV}$ collected during 2016-2018 at the CERN Large Hadron Collider (LHC), corresponding to an integrated luminosity of $137 \mathrm{fb}^{-1}$. The central feature of the CMS experiment is a superconducting solenoid of $6 \mathrm{~m}$ internal diameter providing a magnetic field of $3.8 \mathrm{~T}$. Located within the solenoid volume are a silicon pixel and strip tracker, a lead tungstate crystal electromagnetic calorimeter, and a brass and scintillator hadron calorimeter, each composed of a barrel and two end cap sections. Muons are detected in detectors embedded in the steel flux-return yoke outside the solenoid using three technologies: drift tubes (DTs) in the barrel, cathode strip chambers (CSCs) in the end caps, and resistive-plate chambers (RPCs) in the barrel and end caps. Further details of the CMS detector and the coordinate system definition can be found in Ref. [33].

The CSC detector, which covers a region of pseudorapidity between $|\eta|=0.9$ and 2.4 and plays a critical role for the search described in this Letter, is shown schematically in Fig 1. It is composed of four "stations" in each end cap, labeled ME1 to ME4, which are located approximately $7,8,9.5$, and $10.5 \mathrm{~m}$ away from the interaction point along the beam line axis $(z)$ on both ends of the detector, and are sandwiched between steel absorbers. Each chamber is composed of six thin layers containing cathode strips along the radial direction and anode wires perpendicular to the strips. Charged particles traversing the chambers ionize the gas molecules. The resulting electrons are accelerated towards the anode wires producing an avalanche, while the positive ions travel to the opposite end and induce signals in the cathode strips. By combining the information from signals on the anode wires and the cathode strips of each layer, we can determine the space-time coordinates of each such "hit" with a resolution of $400-500 \mu \mathrm{m}$ and 5 ns [34].

The CMS event reconstruction is based on a particleflow (PF) algorithm [35], which combines information from the tracker, calorimeters, and muon detectors to identify charged and neutral hadrons, photons, electrons, and muons, known collectively as PF candidates, which are clustered into jets using the anti- $k_{\mathrm{T}}$ algorithm with a distance parameter of 0.4 [36-38]. The transverse component of the negative vectorial sum of the momenta of all PF candidates is the missing transverse momentum $\vec{p}_{\mathrm{T}}^{\text {miss }}$, and its magnitude is indicated as $p_{\mathrm{T}}{ }^{\text {miss }}$. The candidate vertex with the largest value of summed physics-object $p_{\mathrm{T}}^{2}$ is taken to be the primary $p p$ interaction vertex. The physics objects are the jets, clustered using the jet finding algorithm $[37,38]$ with the tracks assigned to candidate vertices as inputs, and the associated missing transverse momentum, taken as the negative vector sum of the $p_{\mathrm{T}}$ of those jets. The average neutral energy density from overlapping $p p$ interactions (pileup) is estimated and subtracted from the reconstructed jet energies [39].

The simulated $H \rightarrow S S$ signal samples are generated using POWHEG2.0 [40-43], and include gluon fusion, vector boson fusion, $W H, Z H$, and $t t H$ production modes. The Higgs boson mass is set to $125 \mathrm{GeV}$, while the $S$ mass $\left(m_{S}\right)$ is set to $7,15,40$, or $55 \mathrm{GeV}$. The $c \tau$ is varied between $1 \mathrm{~mm}$ and $100 \mathrm{~m}$. Parton showering, hadronization, and the underlying event are modeled by PYTHIA8.205 and 8.230 [44] with parameters set by the CUETP8M1 [45] and CP5 tunes [46] used for samples simulating the 2016 and 2017/18 datasets, respectively. The NNPDF 3.0 [47] and 3.1 [48] parton distribution functions are used in the generation of all simulated samples. The GEANT4 [49] package is used to model the response of the CMS detector, and simulated minimum-bias events are mixed with the hard interactions in simulated events to match the observed pileup distribution in data.

An LLP that decays after it has traversed the calorimeter systems can produce large $p_{\mathrm{T}}^{\text {miss }}$ because its momentum will remain undetected, as $p_{\mathrm{T}}^{\text {miss }}$ is calculated solely from the tracker and calorimeter information. We exploit this feature by triggering on events with $p_{\mathrm{T}}^{\text {miss }}>120 \mathrm{GeV}$ [50], and subsequently requiring offline $p_{\mathrm{T}}^{\text {miss }}>200 \mathrm{GeV}$. We require at least one jet with $p_{\mathrm{T}}>50 \mathrm{GeV}$ and pseudorapidity $|\eta|<2.4$, because signal events passing the $p_{\mathrm{T}}^{\text {miss }}$ requirement are always produced together with a jet from initial-state radiation. To suppress backgrounds from $W$ boson and top quark production, events containing an isolated electron (muon) passing loose identification criteria [51-53] with $p_{\mathrm{T}}>35$ (25) GeV and $|\eta|<2.5$ (2.4) are vetoed.

The CSC hits are clustered in $\eta$ and the azimuthal angle $\phi$ (in radians) using the DBSCAN algorithm [54], which groups hits by high-density regions. A minimum of 50 hits and a "distance parameter" of 0.2 is used. The geometric 
acceptance for at least one signal LLP of mass 7-55 GeV decaying in the fiducial region of the CSC detectors ranges from $4 \%-27 \%$ for $c \tau$ between $1-10 \mathrm{~m}$, decreasing to $0.5 \%-$ $5 \%$ for $c \tau$ of $100 \mathrm{~m}$. The efficiency for the showers to be reconstructed by the DBSCAN algorithm is approximately $80 \%$ for $b \bar{b}$ and $d \bar{d}$ decays and $65 \%$ for $\tau^{+} \tau^{-}$decays. The accuracy of the simulation prediction for the cluster reconstruction efficiency relies on its ability to model correctly the response of the muon detectors in an environment with multiple particles, each producing a large number of secondary shower particles. This aspect is validated by measuring clusters produced in $Z \rightarrow \mu^{+} \mu^{-}$ data events where one of the muons undergoes bremsstrahlung in the EMD and the associated photon produces an electromagnetic shower, and the associated systematic uncertainty is taken into account in the overall uncertainty on the reconstruction efficiency.

The main SM backgrounds include punch-through jets, muons that undergo bremsstrahlung, and decays of SM LLPs, such as the neutral kaon $K_{L}^{0}$. To suppress background from punch-through jets or muon bremsstrahlung, we reject clusters that have a jet or muon within a $\Delta R=$ $\sqrt{(\Delta \eta)^{2}+(\Delta \phi)^{2}}<0.4$ cone and with jet $p_{\mathrm{T}}>10 \mathrm{GeV}$ or muon $p_{\mathrm{T}}>20 \mathrm{GeV}$. We veto clusters that have any hits in the two innermost rings of the ME1 station (ME1/1 and ME1/2), which have the least absorber material in front, or match any hit [with $\Delta R$ (cluster, hit $)<0.4$ ] in the RPCs located immediately next to ME1/2. In the region where the barrel and end cap muon detectors overlap $(0.8<|\eta|<1.2)$, we veto any cluster matched to any track segment reconstructed in the innermost station of the DT detectors (MB1), or any hit in the RPCs situated in front of and behind MB1 matched to within $\Delta R$ (cluster, segment $/$ hit $)<0.4$. Finally, we reject clusters with $|\eta|>2.0$ to suppress the muon bremsstrahlung background that evaded the muon veto because of the high muon reconstruction and identification inefficiencies at larger $|\eta|$.

The inefficiencies for the jet, ME1, MB1, and RPC hit vetoes are predominantly caused by the presence of pileup particles and random noise, and are measured by randomly sampling the $(\eta, \phi)$ locations of clusters from the signal distribution and evaluating whether a jet or ME1/MB1/RPC hit has been observed within a $\Delta R<0.4$ cone about the cluster's location, using $Z \rightarrow \mu^{+} \mu^{-}$data events with the two muons from the $Z$ decay removed. The loss of efficiency due to the muon veto is also affected by muon segments produced by particles resulting from the LLP decay itself. This contribution is further validated using a control sample of clusters matched to trackless jets made to resemble the signal LLP decay by requiring the neutral energy fraction to be larger than $95 \%$. A $10 \%$ correction is applied to the signal efficiency to account for the simulation's mismodeling of the vetoes. To suppress noncollision backgrounds, we apply filters that remove events containing beam-halo muons or calorimeter noise [55]. To suppress background from cosmic ray muon showers, which produce hits in multiple regions of the CMS detector, we reject any event in which more than a quarter of DT and CSC $\phi$ rings contain 50 or more hits. The efficiency for signal events to pass the ME1/1 and ME1/2 vetoes depends on the LLP decay location, and its average value is between $30 \%-60 \%$ for the signal models considered. The efficiency for the remaining signal to pass all other vetoes is $\sim 80 \%$.

After the veto requirements are applied, the dominant background source consists of decays of SM LLPs, which are predominantly produced by pileup interactions and are independent of the primary interaction that yielded the large $p_{\mathrm{T}}^{\text {miss }}$. These pileup interactions can occur concurrently with the primary interaction (in-time pileup) or in adjacent bunch crossings (out of time or OOT pileup). Clusters produced by OOT pileup are rejected by requiring the cluster time $\left(t_{\text {cluster }}\right)$, defined as the average time of the hits in the cluster relative to the LHC clock, to be consistent with an in-time interaction $\left(-5.0<t_{\text {cluster }}<12.5 \mathrm{~ns}\right)$. A larger time window is used at positive values to capture signal clusters with longer delays from slower moving LLPs. The time window requirement suppresses the background by a factor of 5. An OOT validation region (VR) is defined by selecting events containing clusters with $t_{\text {cluster }} \leq-5 \mathrm{~ns}$, and is used to validate the background prediction method. To reject clusters composed of hits from multiple bunch crossings, we require that the root mean square spread of a cluster's hit times is less than $20 \mathrm{~ns}$.

There are several features that distinguish between signal and background clusters. Clusters from all background processes occur more often at larger values of $|\eta|$, as the effectiveness of the jet and muon vetoes decrease because of increasing reconstruction inefficiencies. Signal clusters often occupy more than one CSC station and occur more frequently in stations further away from the primary interaction point. A cluster identification algorithm was devised that makes successively more restrictive $|\eta|$ requirements as the number of CSC stations containing hits and the distance between the station and the primary interaction point decrease. The cluster identification algorithm has $\sim 80 \%$ efficiency for clusters originating from $S$ decays in the simulation, and suppresses the background by a factor of 3 .

The events that pass the cluster identification criteria are used to define the search region, and those that fail are used as an additional in-time VR. The signal efficiency of the combined cluster reconstruction, veto, and identification selections is shown as a function of the simulated $r$ and $z$ decay positions of the particle $S$ in Fig. 1. The combined efficiency averaged over the full region of the CSC detector is $15 \%-30 \%$ for the signal models considered.

The number of hits in the cluster $\left(N_{\text {hits }}\right)$ and the azimuthal angle between the cluster location and the $\vec{p}_{\mathrm{T}}^{\text {miss }}\left(\Delta \phi_{\mathrm{c}}\right)$ are used to make the final discrimination 
between signal and background. The distribution of $N_{\text {hits }}$ remains high at large $N_{\text {hits }}$ values for signal events, but for background events the distribution of $N_{\text {hits }}$ decreases sharply with increasing $N_{\text {hits }}$ values. For signal, $\Delta \phi_{\mathrm{c}}$ peaks near zero either because the $\vec{p}_{\mathrm{T}}^{\text {miss }}$ results from the same $S$ decay that produced the cluster or the large $p_{\mathrm{T}}^{\text {miss }}$ requirement tends to select highly boosted Higgs bosons for which the $S$ and $H$ momentum vectors are spatially close to each other. For the backgrounds, $\Delta \phi_{c}$ is independent of $N_{\text {hits }}$, enabling the use of the matrix $(A B C D)$ method to predict the background yield in the signal-enriched bin $D$ as $N_{D}=\left(N_{A} N_{C}\right) / N_{B}$, where $N_{X}$ is the background event yield in each bin $X$. Bin $A$ includes events with $\Delta \phi_{c} \geq 0.75$ and $N_{\text {hits }}>130$; bin $B$ includes events with $\Delta \phi_{c} \geq 0.75$ and $N_{\text {hits }} \leq 130$; bin $C$ includes events with $\Delta \phi_{c}<0.75$ and $N_{\text {hits }} \leq 130$; and bin $D$ includes events with $\Delta \phi_{c}<$ 0.75 and $N_{\text {hits }}>130$. The distributions of $N_{\text {hits }}$ in bins $C$ and $D$, and $\Delta \phi_{c}$ in bins $A$ and $D$ are shown in Fig. 2, for the data and for the signal assuming $S \rightarrow d \bar{d}$ decays with various $S$ masses.

To account for a potential signal contribution to bins $A$, $B$, and $C$, a binned maximum likelihood fit is performed simultaneously in the four bins, with a common signal strength parameter scaling the signal yields in each bin. The background component of the fit is constrained to obey the $A B C D$ relationship. Systematic uncertainties that affect the signal yield are missing higher order QCD corrections (21\%), cluster reconstruction and identification efficiency (6\%), veto efficiencies (4\%), jet energy scale (4\%) [56], simulation sample statistical uncertainties $(3 \%-5 \%)$, and luminosity (1.6\%) [57-59]. These systematic uncertainties and the statistical uncertainty of the simulated signal samples are treated as nuisance parameters in the fit.

The background estimation procedure is validated using events in the OOT and in-time VRs, predicting $1.3 \pm 0.6$ and $1.4 \pm 0.6$ events, respectively. In both VRs, 2 events are observed. In the signal-depleted $A, B$, and $C$ bins of the search region, we observe 3,96 , and 47 events in the data, respectively. Using the fit procedure described above and assuming no signal contribution, we predict $2.0 \pm 1.0$ background events in the signal-enriched region $D$, and observe 3 events. The uncertainty in the background prediction is dominated by the statistical uncertainty in the event yields of the signal-depleted $A, B$, and $C$ bins. No excess of events above the SM background is observed.

We evaluate 95\% confidence level (CL) limits on the branching fraction $\mathcal{B}(H \rightarrow S S)$ using the modified frequentist criterion C.L.s [60-62] with the profile likelihood ratio test statistic. The upper limits are shown in Fig. 3 for the $S \rightarrow d \bar{d}$ and $S \rightarrow \tau^{+} \tau^{-}$decay modes, as a function of $c \tau$ for a selection of values of $m_{S}$. The exclusion limits for $S \rightarrow b \bar{b}$ are within $3 \%$ of the exclusion limits for $S \rightarrow d \bar{d}$ for $m_{S}>2 m_{b}$.

In summary, proton-proton collision data at $\sqrt{s}=$ $13 \mathrm{TeV}$ recorded by the CMS experiment in 2016-2018,
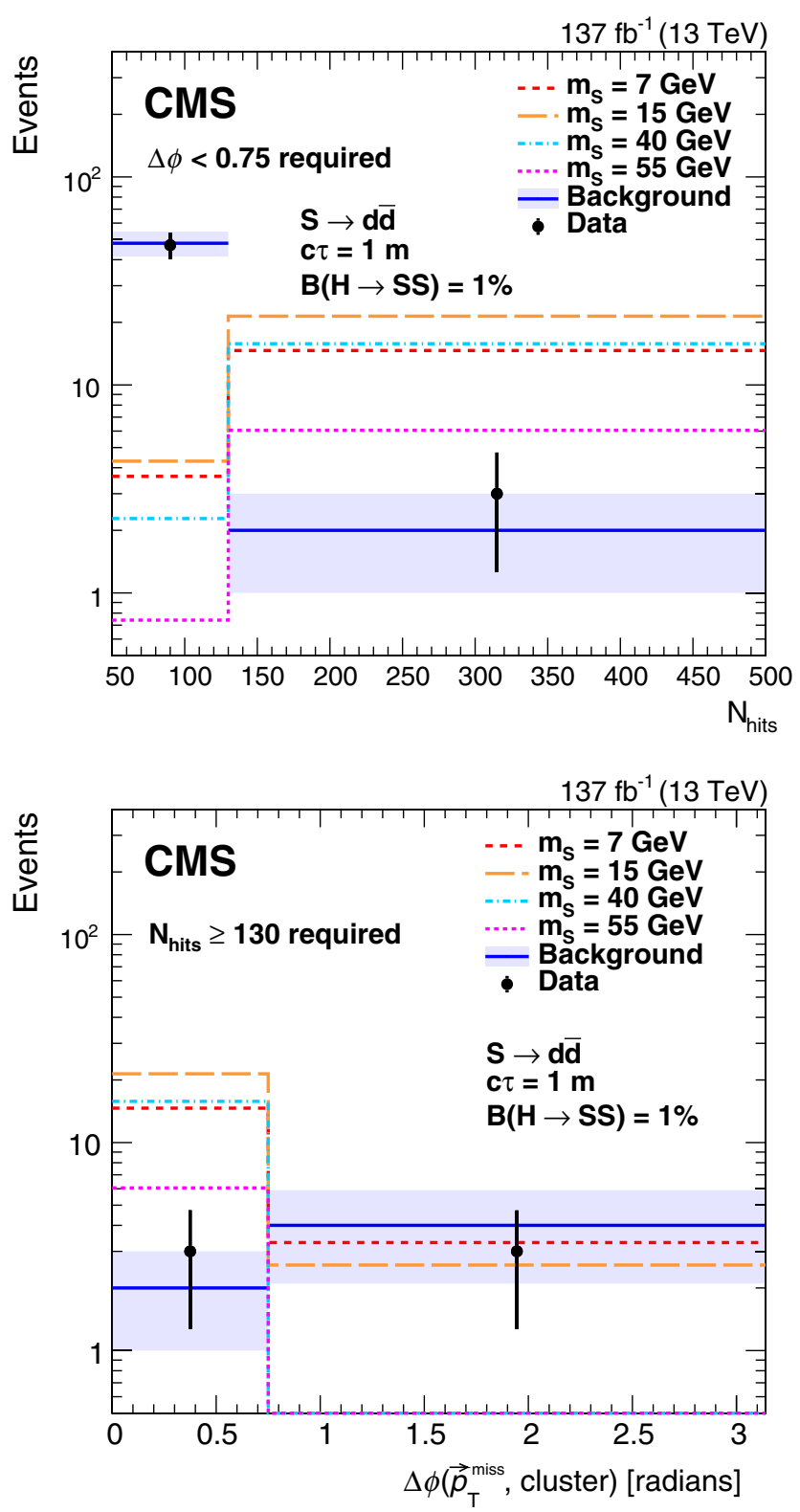

FIG. 2. Distributions of $N_{\text {hits }}$ (left) and $\Delta \phi_{\mathrm{c}}$ (right) in the search region. The background predicted by the fit is shown in blue with the shaded region showing the fitted uncertainty. The expected signal with $\mathcal{B}(H \rightarrow S S)=1 \%, S \rightarrow d \bar{d}$, and $c \tau=1 \mathrm{~m}$ is shown for $m_{S}$ of 7,15, 40, and $55 \mathrm{GeV}$ in various colors and dotted lines. The $N_{\text {hits }}$ distribution includes only events in bins $C$ and $D$, while the $\Delta \phi_{\mathrm{c}}$ includes only events in bins $\mathrm{A}$ and $\mathrm{D}$. The last bin in the $N_{\text {hits }}$ distributions includes overflow events.

corresponding to an integrated luminosity of $137 \mathrm{fb}^{-1}$, have been used to conduct the first search for beyond the standard model (SM) long-lived particles (LLPs) using the CMS end cap muon detectors as a calorimeter. Based on a unique detector signature, the search is largely model independent, with sensitivity to a broad range of LLP decay modes and to LLP masses as low as a few GeV. With the excellent shielding provided by the inner CMS detector, 

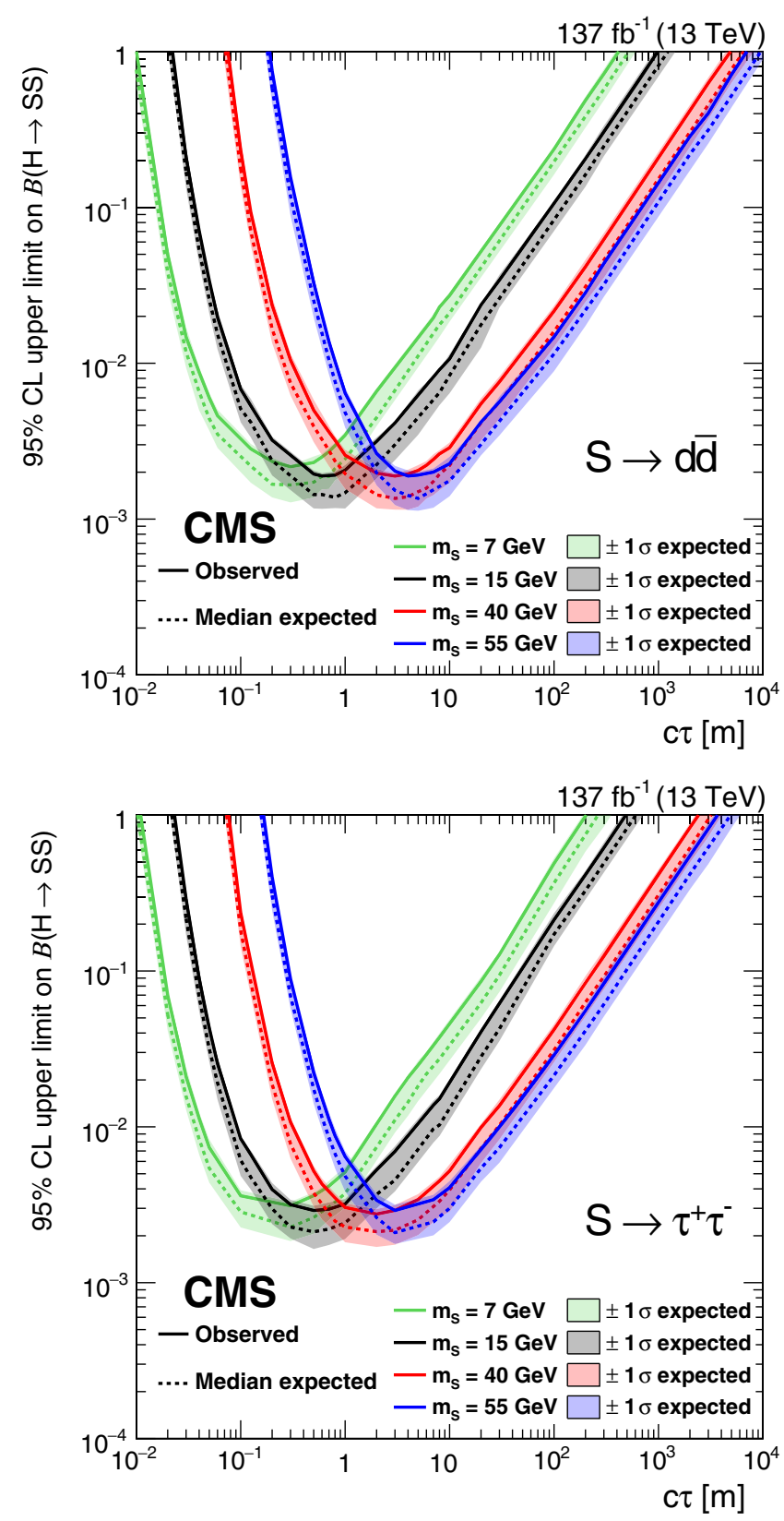

FIG. 3. The 95\% CL expected (dotted curves) and observed (solid curves) upper limits on the branching fraction $\mathcal{B}(H \rightarrow S S$ ) as functions of $c \tau$ for the $S \rightarrow d \bar{d}$ (left) and $S \rightarrow \tau^{+} \tau^{-}$(right) decay modes. The exclusion limits are shown for four different mass hypotheses: $7,15,40$, and $55 \mathrm{GeV}$.

the background is suppressed to a low level and a search for a single LLP decay is possible. No significant deviation from the SM background is observed, and the most stringent limits on the branching fraction of Higgs boson to LLP decaying to $d \bar{d}, b \bar{b}$, and $\tau^{+} \tau^{-}$are set for proper decay lengths $c \tau>6,20$, and $40 \mathrm{~m}$, and LLP masses of 7 , 15 , and $40 \mathrm{GeV}$, respectively. For $c \tau>100 \mathrm{~m}$, this search outperforms the previous best limits [30,31] by a factor of 6 (2) for an LLP mass of $7(\geq 15) \mathrm{GeV}$.
We congratulate our colleagues in the CERN accelerator departments for the excellent performance of the LHC and thank the technical and administrative staffs at CERN and at other CMS institutes for their contributions to the success of the CMS effort. In addition, we gratefully acknowledge the computing centers and personnel of the Worldwide LHC Computing Grid and other centers for delivering so effectively the computing infrastructure essential to our analyses. Finally, we acknowledge the enduring support for the construction and operation of the LHC, the CMS detector, and the supporting computing infrastructure provided by the following funding agencies: BMBWF and FWF (Austria); FNRS and FWO (Belgium); CNPq, CAPES, FAPERJ, FAPERGS, and FAPESP (Brazil); MES (Bulgaria); CERN; CAS, MoST, and NSFC (China); MINCIENCIAS (Colombia); MSES and CSF (Croatia); RIF (Cyprus); SENESCYT (Ecuador); MoER, ERC PUT and ERDF (Estonia); Academy of Finland, MEC, and HIP (Finland); CEA and CNRS/IN2P3 (France); BMBF, DFG, and HGF (Germany); GSRT (Greece); NKFIA (Hungary); DAE and DST (India); IPM (Iran); SFI (Ireland); INFN (Italy); MSIP and NRF (Republic of Korea); MES (Latvia); LAS (Lithuania); MOE and UM (Malaysia); BUAP, CINVESTAV, CONACYT, LNS, SEP, and UASLP-FAI (Mexico); MOS (Montenegro); MBIE (New Zealand); PAEC (Pakistan); MSHE and NSC (Poland); FCT (Portugal); JINR (Dubna); MON, RosAtom, RAS, RFBR, and NRC KI (Russia); MESTD (Serbia); SEIDI, CPAN, PCTI, and FEDER (Spain); MOSTR (Sri Lanka); Swiss Funding Agencies (Switzerland); MST (Taipei); ThEPCenter, IPST, STAR, and NSTDA (Thailand); TUBITAK and TAEK (Turkey); NASU (Ukraine); STFC (United Kingdom); DOE and NSF (USA).

[1] G. F. Giudice and A. Romanino, Split supersymmetry, Nucl. Phys. B699, 65 (2004); Erratum, Nucl. Phys. B706, 487 (2005).

[2] J. L. Hewett, B. Lillie, M. Masip, and T. G. Rizzo, Signatures of long-lived gluinos in split supersymmetry, J. High Energy Phys. 09 (2004) 070.

[3] N. Arkani-Hamed, S. Dimopoulos, G. F. Giudice, and A. Romanino, Aspects of split supersymmetry, Nucl. Phys. B709, 3 (2005).

[4] P. Gambino, G. F. Giudice, and P. Slavich, Gluino decays in split supersymmetry, Nucl. Phys. B726, 35 (2005).

[5] A. Arvanitaki, N. Craig, S. Dimopoulos, and G. Villadoro, Mini-split, J. High Energy Phys. 02 (2013) 126.

[6] N. Arkani-Hamed, A. Gupta, D. E. Kaplan, N. Weiner, and T. Zorawski, Simply unnatural supersymmetry, arXiv: 1212.6971.

[7] P. Fayet, Supergauge invariant extension of the Higgs mechanism and a model for the electron and its neutrino, Nucl. Phys. B90, 104 (1975). 
[8] G. R. Farrar and P. Fayet, Phenomenology of the production, decay, and detection of new hadronic states associated with supersymmetry, Phys. Lett. 76B, 575 (1978).

[9] S. Weinberg, Supersymmetry at ordinary energies. Masses and conservation laws, Phys. Rev. D 26, 287 (1982).

[10] R. Barbier, C. Bérat, M. Besançon, M. Chemtob, A. Deandrea, E. Dudas, P. Fayet, S. Lavignac, G. Moreau, E. Perez, and Y. Sirois, $R$-parity violating supersymmetry, Phys. Rep. 420, 1 (2005).

[11] G. F. Giudice and R. Rattazzi, Theories with gauge mediated supersymmetry breaking, Phys. Rep. 322, 419 (1999).

[12] P. Meade, N. Seiberg, and D. Shih, General gauge mediation, Prog. Theor. Phys. Suppl. 177, 143 (2009).

[13] M. Buican, P. Meade, N. Seiberg, and D. Shih, Exploring general gauge mediation, J. High Energy Phys. 03 (2009) 016.

[14] J. Fan, M. Reece, and J. T. Ruderman, Stealth supersymmetry, J. High Energy Phys. 11 (2011) 012.

[15] J. Fan, M. Reece, and J. T. Ruderman, A stealth supersymmetry sampler, J. High Energy Phys. 07 (2012) 196.

[16] M. J. Strassler and K. M. Zurek, Echoes of a hidden valley at hadron colliders, Phys. Lett. B 651, 374 (2007).

[17] M. J. Strassler and K. M. Zurek, Discovering the Higgs through highly-displaced vertices, Phys. Lett. B 661, 263 (2008).

[18] T. Han, Z. Si, K. M. Zurek, and M. J. Strassler, Phenomenology of hidden valleys at hadron colliders, J. High Energy Phys. 07 (2008) 008.

[19] Y. Cui, L. Randall, and B. Shuve, A WIMPy baryogenesis miracle, J. High Energy Phys. 04 (2012) 075.

[20] Y. Cui and R. Sundrum, Baryogenesis for weakly interacting massive particles, Phys. Rev. D 87, 116013 (2013).

[21] Y. Cui and B. Shuve, Probing baryogenesis with displaced vertices at the LHC, J. High Energy Phys. 02 (2015) 049.

[22] D. Smith and N. Weiner, Inelastic dark matter, Phys. Rev. D 64, 043502 (2001).

[23] Z. Chacko, H.-S. Goh, and R. Harnik, Natural Electroweak Breaking from a Mirror Symmetry, Phys. Rev. Lett. 96, 231802 (2006).

[24] D. Curtin and C. B. Verhaaren, Discovering uncolored naturalness in exotic Higgs decays, J. High Energy Phys. 12 (2015) 072.

[25] H.-C. Cheng, S. Jung, E. Salvioni, and Y. Tsai, Exotic quarks in twin Higgs models, J. High Energy Phys. 03 (2016) 074.

[26] N. Craig, A. Katz, M. Strassler, and R. Sundrum, Naturalness in the dark at the LHC, J. High Energy Phys. 07 (2015) 105.

[27] M. J. Strassler, On the phenomenology of hidden valleys with heavy flavor, arXiv:0806.2385.

[28] J. E. Juknevich, D. Melnikov, and M. J. Strassler, A pureglue hidden valley I. states and decays, J. High Energy Phys. 07 (2009) 055.

[29] CMS Collaboration, Search for long-lived particles using displaced jets in proton-proton collisions at $\sqrt{s}=13 \mathrm{TeV}$, Phys. Rev. D 104, 012015 (2021).

[30] ATLAS Collaboration, Search for long-lived particles produced in $p p$ collisions at $\sqrt{s}=13 \mathrm{TeV}$ that decay into displaced hadronic jets in the ATLAS muon spectrometer, Phys. Rev. D 99, 052005 (2019).
[31] ATLAS Collaboration, Search for long-lived neutral particles produced in $p p$ collisions at $\sqrt{s}=13 \mathrm{TeV}$ decaying into displaced hadronic jets in the ATLAS inner detector and muon spectrometer, Phys. Rev. D 101, 052013 (2020).

[32] HEPData record for this analysis (2021), http://dx.doi.org/ 10.17182/hepdata.104408.

[33] CMS Collaboration, The CMS experiment at the CERN LHC, J. Instrum. 3, S08004 (2008).

[34] CMS Collaboration, Performance of the CMS cathode strip chambers with cosmic rays, J. Instrum. 5, T03018 (2010).

[35] CMS Collaboration, Particle-flow reconstruction and global event description with the CMS detector, J. Instrum. 12, P10003 (2017).

[36] M. Cacciari and G. P. Salam, Dispelling the $N^{3}$ myth for the $k_{\mathrm{T}}$ jet-finder, Phys. Lett. B 641, 57 (2006).

[37] M. Cacciari, G. P. Salam, and G. Soyez, The anti- $k_{\mathrm{T}}$ jet clustering algorithm, J. High Energy Phys. 04 (2008) 063.

[38] M. Cacciari, G. P. Salam, and G. Soyez, FAStJet user manual, Eur. Phys. J. C 72, 1896 (2012).

[39] M. Cacciari and G. P. Salam, Pileup subtraction using jet areas, Phys. Lett. B 659, 119 (2008).

[40] P. Nason, A new method for combining NLO QCD with shower Monte Carlo algorithms, J. High Energy Phys. 11 (2004) 040.

[41] S. Frixione, P. Nason, and C. Oleari, Matching NLO QCD computations with parton shower simulations: The POWHEG method, J. High Energy Phys. 11 (2007) 070.

[42] S. Alioli, P. Nason, C. Oleari, and E. Re, A general framework for implementing NLO calculations in shower Monte Carlo programs: The POWHEG BOX, J. High Energy Phys. 06 (2010) 043.

[43] E. Re, Single-top Wt-channel production matched with parton showers using the POWHEG method, Eur. Phys. J. C 71, 1547 (2011).

[44] T. Sjöstrand, S. Ask, J. R. Christiansen, R. Corke, N. Desai, P. Ilten, S. Mrenna, S. Prestel, C. O. Rasmussen, and P.Z. Skands, An introduction to PYTHIA 8.2, Comput. Phys. Commun. 191, 159 (2015).

[45] CMS Collaboration, Event generator tunes obtained from underlying event and multiparton scattering measurements, Eur. Phys. J. C 76, 155 (2016).

[46] CMS Collaboration, Extraction and validation of a new set of CMS PYTHIA8 tunes from underlying-event measurements, Eur. Phys. J. C 80, 4 (2020).

[47] R. D. Ball et al. (NNPDF Collaboration), Parton distributions for the LHC Run II, J. High Energy Phys. 04 (2015) 040.

[48] R. D. Ball et al. (NNPDF Collaboration), Parton distributions from high-precision collider data, Eur. Phys. J. C 77, 663 (2017).

[49] S. Agostinelli et al. (GEANT4 Collaboration), GEANT4-a simulation toolkit, Nucl. Instrum. Methods Phys. Res., Sect. A 506, 250 (2003).

[50] CMS Collaboration, The CMS trigger system, J. Instrum. 12, P01020 (2017).

[51] CMS Collaboration, Electron and photon reconstruction and identification with the CMS experiment at the CERN LHC, J. Instrum. 16, P05014 (2021). 
[52] CMS Collaboration, Performance of the CMS muon detector and muon reconstruction with proton-proton collisions at $\sqrt{s}=13 \mathrm{TeV}$, J. Instrum. 13, P06015 (2018).

[53] CMS Collaboration, Performance of the reconstruction and identification of high-momentum muons in proton-proton collisions at $\sqrt{s}=13 \mathrm{TeV}$, J. Instrum. 15, P02027 (2020).

[54] M. Ester, H.-P. Kriegel, J. Sander, and X. Xu, A densitybased algorithm for discovering clusters in large spatial databases with noise, in Proceedings of the Second International Conference on Knowledge Discovery and Data Mining (Association for the Advancement of Artificial Intelligence, 1996), p. 226, https://www.aaai.org/Papers/ KDD/1996/KDD96-037.pdf.

[55] CMS Collaboration, Missing transverse energy performance of the CMS detector, J. Instrum. 6, P09001 (2011).

[56] CMS Collaboration, Jet energy scale and resolution in the CMS experiment in pp collisions at $8 \mathrm{TeV}$, J. Instrum. 12, P02014 (2017).

[57] CMS Collaboration, CMS luminosity measurements for the 2016 data-taking period, CMS Physics Analysis Summary,
CMS-PAS-LUM-17-001, 2016, https://cds.cern.ch/record/ 2257069.

[58] CMS Collaboration, CMS luminosity measurements for the 2017 data-taking period at $\sqrt{s}=13 \mathrm{TeV}$, CMS Physics Analysis Summary, CMS-PAS-LUM-17-004, 2017, https:// cds.cern.ch/record/2621960.

[59] CMS Collaboration, CMS luminosity measurements for the 2018 data-taking period at $\sqrt{s}=13 \mathrm{TeV}, \mathrm{CMS}$ Physics Analysis Summary, CMS-PAS-LUM-18-002, 2018, https://cds.cern.ch/record/2676164.

[60] T. Junk, Confidence level computation for combining searches with small statistics, Nucl. Instrum. Methods Phys. Res., Sect. A 434, 435 (1999).

[61] A. L. Read, Presentation of search results: the $\mathrm{CL}_{\mathrm{s}}$ technique, J. Phys. G 28, 2693 (2002).

[62] The ATLAS and The CMS Collaborations, The LHC Higgs Combination Group, Procedure for the LHC Higgs boson search combination in Summer 2011, Technical Report, CMS-NOTE-2011-005, ATL-PHYS-PUB-201111, 2011, https://cds.cern.ch/record/1379837.

A. Tumasyan, ${ }^{1}$ W. Adam, ${ }^{2}$ J. W. Andrejkovic, ${ }^{2}$ T. Bergauer, ${ }^{2}$ S. Chatterjee, ${ }^{2}$ M. Dragicevic, ${ }^{2}$ A. Escalante Del Valle, ${ }^{2}$ R. Frühwirth, ${ }^{2, b}$ M. Jeitler, ${ }^{2, b}$ N. Krammer, ${ }^{2}$ L. Lechner, ${ }^{2}$ D. Liko, ${ }^{2}$ I. Mikulec, ${ }^{2}$ P. Paulitsch, ${ }^{2}$ F. M. Pitters, ${ }^{2}$ J. Schieck, ${ }^{2, b}$ R. Schöfbeck, ${ }^{2}$ D. Schwarz, ${ }^{2}$ S. Templ, ${ }^{2}$ W. Waltenberger, ${ }^{2}$ C.-E. Wulz, ${ }^{2, b}$ V. Chekhovsky, ${ }^{3}$ A. Litomin, ${ }^{3}$ V. Makarenko, ${ }^{3}$ M. R. Darwish, ${ }^{4, c}$ E. A. De Wolf, ${ }^{4}$ T. Janssen, ${ }^{4}$ T. Kello, ${ }^{4, d}$ A. Lelek, ${ }^{4}$ H. Rejeb Sfar, ${ }^{4}$ P. Van Mechelen, ${ }^{4}$ S. Van Putte, ${ }^{4}$ N. Van Remortel, ${ }^{4}$ F. Blekman, ${ }^{5}$ E. S. Bols, ${ }^{5}$ J. D'Hondt, ${ }^{5}$ M. Delcourt, ${ }^{5}$ H. El Faham, ${ }^{5}$ S. Lowette, ${ }^{5}$ S. Moortgat, ${ }^{5}$ A. Morton, ${ }^{5}$ D. Müller, ${ }^{5}$ A. R. Sahasransu, ${ }^{5}$ S. Tavernier, ${ }^{5}$ W. Van Doninck, ${ }^{5}$ P. Van Mulders, ${ }^{5}$ D. Beghin, ${ }^{6}$ B. Bilin, ${ }^{6}$ B. Clerbaux, ${ }^{6}$ G. De Lentdecker, ${ }^{6}$ L. Favart, ${ }^{6}$ A. Grebenyuk, ${ }^{6}$ A. K. Kalsi, ${ }^{6}$ K. Lee, ${ }^{6}$ M. Mahdavikhorrami, ${ }^{6}$ I. Makarenko, ${ }^{6}$ L. Moureaux, ${ }^{6}$ L. Pétré, ${ }^{6}$ A. Popov, ${ }^{6}$ N. Postiau, ${ }^{6}$ E. Starling, ${ }^{6}$ L. Thomas, ${ }^{6}$ M. Vanden Bemden, ${ }^{6}$ C. Vander Velde, ${ }^{6}$ P. Vanlaer, ${ }^{6}$ L. Wezenbeek, ${ }^{6}$ T. Cornelis, ${ }^{7}$ D. Dobur, ${ }^{7}$ J. Knolle, ${ }^{7}$ L. Lambrecht, ${ }^{7}$ G. Mestdach, ${ }^{7}$ M. Niedziela, ${ }^{7}$ C. Roskas, ${ }^{7}$ A. Samalan, ${ }^{7}$ K. Skovpen, ${ }^{7}$ M. Tytgat,${ }^{7}$ B. Vermassen, ${ }^{7}$ M. Vit, ${ }^{7}$ A. Benecke, ${ }^{8}$ A. Bethani, ${ }^{8}$ G. Bruno, ${ }^{8}$ F. Bury, ${ }^{8}$ C. Caputo, ${ }^{8}$ P. David, ${ }^{8}$ C. Delaere, ${ }^{8}$ I. S. Donertas, ${ }^{8}$ A. Giammanco, ${ }^{8}$ K. Jaffel,${ }^{8}$ Sa. Jain, ${ }^{8}$ V. Lemaitre, ${ }^{8}$ K. Mondal, ${ }^{8}$ J. Prisciandaro, ${ }^{8}$ A. Taliercio, ${ }^{8}$ M. Teklishyn, ${ }^{8}$ T. T. Tran, ${ }^{8}$ P. Vischia, ${ }^{8}$ S. Wertz, ${ }^{8}$ G. A. Alves,${ }^{9}$ C. Hensel,,${ }^{9}$ A. Moraes, ${ }^{9}$ W. L. Aldá Júnior, ${ }^{10}$ M. Alves Gallo Pereira, ${ }^{10}$ M. Barroso Ferreira Filho, ${ }^{10}$ H. Brandao Malbouisson, ${ }^{10}$ W. Carvalho, ${ }^{10}$ J. Chinellato, ${ }^{10,}$

E. M. Da Costa, ${ }^{10}$ G. G. Da Silveira, ${ }^{10, \mathrm{f}}$ D. De Jesus Damiao, ${ }^{10}$ S. Fonseca De Souza, ${ }^{10}$ D. Matos Figueiredo, ${ }^{10}$ C. Mora Herrera, ${ }^{10}$ K. Mota Amarilo, ${ }^{10}$ L. Mundim, ${ }^{10}$ H. Nogima, ${ }^{10}$ P. Rebello Teles,${ }^{10}$ A. Santoro, ${ }^{10}$

S. M. Silva Do Amaral, ${ }^{10}$ A. Sznajder, ${ }^{10}$ M. Thiel, ${ }^{10}$ F. Torres Da Silva De Araujo, ${ }^{10}$ A. Vilela Pereira, ${ }^{10}$ C. A. Bernardes, ${ }^{11 a, f}$ L. Calligaris, ${ }^{11 \mathrm{a}}$ T. R. Fernandez Perez Tomei, ${ }^{11 \mathrm{a}}$ E. M. Gregores, ${ }^{11 \mathrm{a}, 1 \mathrm{~b}}$ D. S. Lemos, ${ }^{1 \mathrm{la}}$ P. G. Mercadante, ${ }^{11 \mathrm{a}, 1 \mathrm{~b}}$

S. F. Novaes, ${ }^{11 a}$ Sandra S. Padula, ${ }^{11 a}$ A. Aleksandrov, ${ }^{12}$ G. Antchev, ${ }^{12}$ R. Hadjiiska, ${ }^{12}$ P. Iaydjiev, ${ }^{12}$ M. Misheva, ${ }^{12}$ M. Rodozov, ${ }^{12}$ M. Shopova, ${ }^{12}$ G. Sultanov, ${ }^{12}$ A. Dimitrov, ${ }^{13}$ T. Ivanov, ${ }^{13}$ L. Litov, ${ }^{13}$ B. Pavlov, ${ }^{13}$ P. Petkov, ${ }^{13}$ A. Petrov, ${ }^{13}$ T. Cheng, ${ }^{14}$ T. Javaid, ${ }^{14, \mathrm{~g}}$ M. Mittal, ${ }^{14}$ L. Yuan, ${ }^{14}$ M. Ahmad, ${ }^{15}$ G. Bauer, ${ }^{15}$ C. Dozen,,${ }^{15}$ Z. Hu, ${ }^{15}$ J. Martins, ${ }^{15, \mathrm{i}}$ Y. Wang, ${ }^{15}$ K. Yi, ${ }^{15, j, k}$ E. Chapon, ${ }^{16}$ G. M. Chen, ${ }^{16, g}$ H. S. Chen, ${ }^{16, g}$ M. Chen, ${ }^{16}$ F. Iemmi, ${ }^{16}$ A. Kapoor, ${ }^{16}$ D. Leggat, ${ }^{16}$ H. Liao, ${ }^{16}$ Z.-A. Liu, ${ }^{16,1}$ V. Milosevic, ${ }^{16}$ F. Monti, ${ }^{16}$ R. Sharma, ${ }^{16}$ J. Tao, ${ }^{16}$ J. Thomas-wilsker, ${ }^{16}$ J. Wang, ${ }^{16}$ H. Zhang, ${ }^{16}$ J. Zhao, ${ }^{16}$ A. Agapitos, ${ }^{17}$ Y. An,${ }^{17}$ Y. Ban,,${ }^{17}$ C. Chen, ${ }^{17}$ A. Levin, ${ }^{17}$ Q. Li, ${ }^{17}$ X. Lyu, ${ }^{17}$ Y. Mao, ${ }^{17}$ S. J. Qian, ${ }^{17}$ D. Wang, ${ }^{17}$ Q. Wang, ${ }^{17}$ J. Xiao, ${ }^{17}$ M. Lu, ${ }^{18}$ Z. You, ${ }^{18}$ X. Gao,${ }^{19,}$ H. Okawa, ${ }^{19}$ Z. Lin,${ }^{20}$ M. Xiao, ${ }^{20}$ C. Avila, ${ }^{21}$ A. Cabrera, ${ }^{21}$ C. Florez,${ }^{21}$ J. Fraga, ${ }^{21}$ J. Mejia Guisao, ${ }^{22}$ F. Ramirez, ${ }^{22}$ J. D. Ruiz Alvarez, ${ }^{22}$ C. A. Salazar González, ${ }^{22}$ D. Giljanovic, ${ }^{23}$ N. Godinovic, ${ }^{23}$ D. Lelas, ${ }^{23}$ I. Puljak, ${ }^{23}$ Z. Antunovic, ${ }^{24}$ M. Kovac, ${ }^{24}$ T. Sculac, ${ }^{24}$ V. Brigljevic, ${ }^{25}$ D. Ferencek, ${ }^{25}$ D. Majumder, ${ }^{25}$ M. Roguljic, ${ }^{25}$ A. Starodumov, ${ }^{25, \mathrm{~m}}$ T. Susa, ${ }^{25}$ A. Attikis, ${ }^{26}$ K. Christoforou, ${ }^{26}$ E. Erodotou, ${ }^{26}$ A. Ioannou, ${ }^{26}$ G. Kole, ${ }^{26}$ M. Kolosova, ${ }^{26}$ S. Konstantinou, ${ }^{26}$ J. Mousa, ${ }^{26}$ C. Nicolaou, ${ }^{26}$ F. Ptochos,${ }^{26}$ P. A. Razis, ${ }^{26}$ H. Rykaczewski, ${ }^{26}$ H. Saka, ${ }^{26}$ M. Finger, ${ }^{27, n}$ M. Finger Jr., ${ }^{27, n}$ A. Kveton, ${ }^{27}$ E. Ayala, ${ }^{28}$ E. Carrera Jarrin, ${ }^{29}$ A. A. Abdelalim, ${ }^{30, o p}$ S. Elgammal,,${ }^{30, q}$ M. A. Mahmoud, ${ }^{31}$ 
Y. Mohammed, ${ }^{31}$ S. Bhowmik, ${ }^{32}$ R. K. Dewanjee, ${ }^{32}$ K. Ehataht, ${ }^{32}$ M. Kadastik, ${ }^{32}$ S. Nandan, ${ }^{32}$ C. Nielsen, ${ }^{32}$ J. Pata, ${ }^{32}$ M. Raidal, ${ }^{32}$ L. Tani,${ }^{32}$ C. Veelken, ${ }^{32}$ P. Eerola ${ }^{33}$ L. Forthomme, ${ }^{33}$ H. Kirschenmann, ${ }^{33}$ K. Osterberg, ${ }^{33}$ M. Voutilainen, ${ }^{33}$ S. Bharthuar, ${ }^{34}$ E. Brücken, ${ }^{34}$ F. Garcia,${ }^{34}$ J. Havukainen, ${ }^{34}$ M. S. Kim,,${ }^{34}$ R. Kinnunen, ${ }^{34}$ T. Lampén,${ }^{34}$ K. Lassila-Perinin, ${ }^{34}$ S. Lehti, ${ }^{34}$ T. Lindén, ${ }^{34}$ M. Lotti, ${ }^{34}$ L. Martikainen, ${ }^{34}$ M. Myllymäki, ${ }^{34}$ J. Ott, ${ }^{34}$ H. Siikonen, ${ }^{34}$ E. Tuominen, ${ }^{34}$ J. Tuominiemi, ${ }^{34}$ P. Luukka, ${ }^{35}$ H. Petrow, ${ }^{35}$ T. Tuuva, ${ }^{35}$ C. Amendola ${ }^{36}$ M. Besancon, ${ }^{36}$ F. Couderc, ${ }^{36}$ M. Dejardin, ${ }^{36}$ D. Denegri, ${ }^{36}$ J. L. Faure, ${ }^{36}$ F. Ferri, ${ }^{36}$ S. Ganjour, ${ }^{36}$ A. Givernaud,${ }^{36}$ P. Gras, ${ }^{36}$ G. Hamel de Monchenault, ${ }^{36}$ P. Jarry, ${ }^{36}$ B. Lenzi, ${ }^{36}$ E. Locci, ${ }^{36}$ J. Malcles, ${ }^{36}$ J. Rander, ${ }^{36}$ A. Rosowsky, ${ }^{36}$ M. Ö. Sahin, ${ }^{36}$ A. Savoy-Navarro, ${ }^{36, r}$ M. Titov, ${ }^{36}$ G. B. Yu, ${ }^{36}$ S. Ahuja, ${ }^{37}$ F. Beaudette, ${ }^{37}$ M. Bonanomi, ${ }^{37}$ A. Buchot Perraguin, ${ }^{37}$ P. Busson, ${ }^{37}$ A. Cappati, ${ }^{37}$ C. Charlot,${ }^{37}$ O. Davignon, ${ }^{37}$ B. Diab,${ }^{37}$ G. Falmagne, ${ }^{37}$ S. Ghosh,${ }^{37}$ R. Granier de Cassagnac,${ }^{37}$ A. Hakimi,${ }^{37}$ I. Kucher, ${ }^{37}$ J. Motta, ${ }^{37}$ M. Nguyen, ${ }^{37}$ C. Ochando, ${ }^{37}$ P. Paganini, ${ }^{37}$ J. Rembser, ${ }^{37}$ R. Salerno, ${ }^{37}$ U. Sarkar, ${ }^{37}$ J. B. Sauvan, ${ }^{37}$ Y. Sirois,${ }^{37}$ A. Tarabini, ${ }^{37}$ A. Zabi, ${ }^{37}$ A. Zghiche, ${ }^{37}$ J.-L. Agram, ${ }^{38, s}$ J. Andrea, ${ }^{38}$ D. Apparu, ${ }^{38}$ D. Bloch, ${ }^{38}$ G. Bourgatte, ${ }^{38}$ J.-M. Brom, ${ }^{38}$ E. C. Chabert ${ }^{38}$ C. Collard, ${ }^{38}$ D. Darej, ${ }^{38}$ J.-C. Fontaine, ${ }^{38, \mathrm{~s}}$ U. Goerlach,${ }^{38}$ C. Grimault, ${ }^{38}$ A.-C. Le Bihan ${ }^{38}$ E. Nibigira, ${ }^{38}$ P. Van Hove ${ }^{38}$ E. Asilar, ${ }^{39}$ S. Beauceron,${ }^{39}$ C. Bernet, ${ }^{39}$ G. Boudoul,${ }^{39}$ C. Camen, ${ }^{39}$ A. Carle, ${ }^{39}$ N. Chanon,${ }^{39}$ D. Contardo, ${ }^{39}$ P. Depasse, ${ }^{39}$ H. El Mamouni, ${ }^{39}$ J. Fay, ${ }^{39}$ S. Gascon, ${ }^{39}$ M. Gouzevitch, ${ }^{39}$ B. Ille, ${ }^{39}$ I. B. Laktineh, ${ }^{39}$ H. Lattaud, ${ }^{39}$ A. Lesauvage, ${ }^{39}$ M. Lethuillier, ${ }^{39}$ L. Mirabito, ${ }^{39}$ S. Perries, ${ }^{39}$ K. Shchablo, ${ }^{39}$ V. Sordini,${ }^{39}$ L. Torterotot, ${ }^{39}$ G. Touquet, ${ }^{39}$ M. Vander Donckt, ${ }^{39}$ S. Viret, ${ }^{39}$ A. Khvedelidze, ${ }^{40, n}$ I. Lomidze, ${ }^{40}$ Z. Tsamalaidze, ${ }^{40, n}$ V. Botta, ${ }^{41}$ L. Feld, ${ }^{41}$ K. Klein, ${ }^{41}$ M. Lipinski, ${ }^{41}$ D. Meuser, ${ }^{41}$ A. Pauls,${ }^{41}$ N. Röwert ${ }^{41}$ J. Schulz,${ }^{41}$ M. Teroerde,${ }^{41}$ A. Dodonova, ${ }^{42}$ D. Eliseev, ${ }^{42}$ M. Erdmann, ${ }^{42}$ P. Fackeldey, ${ }^{42}$ B. Fischer, ${ }^{42}$ S. Ghosh, ${ }^{42}$ T. Hebbeker, ${ }^{42}$ K. Hoepfner, ${ }^{42}$ F. Ivone, ${ }^{42}$ L. Mastrolorenzo, ${ }^{42}$ M. Merschmeyer, ${ }^{42}$ A. Meyer, ${ }^{42}$ G. Mocellin, ${ }^{42}$ S. Mondal, ${ }^{42}$ S. Mukherjee, ${ }^{42}$ D. Noll, ${ }^{42}$ A. Novak, ${ }^{42}$ T. Pook, ${ }^{42}$ A. Pozdnyakov, ${ }^{42}$ Y. Rath, ${ }^{42}$ H. Reithler, ${ }^{42}$ J. Roemer, ${ }^{42}$ A. Schmidt, ${ }^{42}$ S. C. Schuler, ${ }^{42}$ A. Sharma, ${ }^{42}$ L. Vigilante, ${ }^{42}$ S. Wiedenbeck, ${ }^{42}$ S. Zaleski, ${ }^{42}$ C. Dziwok, ${ }^{43}$ G. Flügge, ${ }^{43}$ W. Haj Ahmad, ${ }^{43, t}$ O. Hlushchenko, ${ }^{43}$ T. Kress, ${ }^{43}$ A. Nowack, ${ }^{43}$ C. Pistone, ${ }^{43}$ O. Pooth, ${ }^{43}$ D. Roy, ${ }^{43}$ H. Sert, ${ }^{43}$ A. Stahl, ${ }^{43, u}$ T. Ziemons, ${ }^{43}$ A. Zotz,${ }^{43}$ H. Aarup Petersen, ${ }^{44}$ M. Aldaya Martin, ${ }^{44}$ P. Asmuss, ${ }^{44}$ S. Baxter ${ }^{44}$ M. Bayatmakou, ${ }^{44}$ O. Behnke, ${ }^{44}$ A. Bermúdez Martínez,${ }^{44}$ S. Bhattacharya, ${ }^{44}$ A. A. Bin Anuar, ${ }^{44}$ K. Borras, ${ }^{44, v}$ D. Brunner, ${ }^{44}$ A. Campbell, ${ }^{44}$ A. Cardini,${ }^{44}$ C. Cheng ${ }^{44}$ F. Colombina, ${ }^{44}$ S. Consuegra Rodríguez, ${ }^{44}$ G. Correia Silva, ${ }^{44}$ V. Danilov, ${ }^{44}$ M. De Silva, ${ }^{44}$ L. Didukh, ${ }^{44}$ G. Eckerlin, ${ }^{44}$ D. Eckstein, ${ }^{44}$ L. I. Estevez Banos, ${ }^{44}$ O. Filatov, ${ }^{44}$ E. Gallo, ${ }^{44, w}$ A. Geiser, ${ }^{44}$ A. Giraldi, ${ }^{44}$ A. Grohsjean, ${ }^{44}$ M. Guthoff, ${ }^{44}$ A. Jafari, ${ }^{44, x}$ N. Z. Jomhari, ${ }^{44}$ H. Jung, ${ }^{44}$ A. Kasem ${ }^{44, v}$ M. Kasemann, ${ }^{44}$ H. Kaveh,${ }^{44}$ C. Kleinwort, ${ }^{44}$ D. Krücker, ${ }^{44}$ W. Lange, ${ }^{44}$ J. Lidrych, ${ }^{44}$ K. Lipka ${ }^{44}$ W. Lohmann, ${ }^{44, y}$ R. Mankel, ${ }^{44}$ I.-A. Melzer-Pellmann, ${ }^{44}$ M. Mendizabal Morentin, ${ }^{44}$ J. Metwally, ${ }^{44}$ A. B. Meyer, ${ }^{44}$ M. Meyer, ${ }^{44}$ J. Mnich, ${ }^{44}$ A. Mussgiller, ${ }^{44}$ Y. Otarid, ${ }^{44}$ D. Pérez Adán, ${ }^{44}$ D. Pitzl, ${ }^{44}$ A. Raspereza, ${ }^{44}$ B. Ribeiro Lopes ${ }^{44}$ J. Rübenach, ${ }^{44}$ A. Saggio, ${ }^{44}$ A. Saibel, ${ }^{44}$ M. Savitskyi, ${ }^{44}$ M. Scham, ${ }^{44, z}$ V. Scheurer, ${ }^{44}$ P. Schütze, ${ }^{44}$ C. Schwanenberger, ${ }^{44, w}$ A. Singh, ${ }^{44}$ R. E. Sosa Ricardo, ${ }^{44}$ D. Stafford, ${ }^{44}$ N. Tonon, ${ }^{44}$ M. Van De Klundert, ${ }^{44}$ R. Walsh,${ }^{44}$ D. Walter,${ }^{44}$ Y. Wen, ${ }^{44}$ K. Wichmann, ${ }^{44}$ L. Wiens, ${ }^{44}$ C. Wissing, ${ }^{44}$ S. Wuchterl, ${ }^{44}$ R. Aggleton, ${ }^{45}$ S. Albrecht,${ }^{45}$ S. Bein, ${ }^{45}$ L. Benato, ${ }^{45}$ P. Connor, ${ }^{45}$ K. De Leo, ${ }^{45}$ M. Eich ${ }^{45}$ F. Feindt ${ }^{45}$ A. Fröhlich,${ }^{45}$ C. Garbers ${ }^{45}$ E. Garutti, ${ }^{45}$ P. Gunnellini, ${ }^{45}$ M. Hajheidari, ${ }^{45}$ J. Haller,${ }^{45}$ A. Hinzmann, ${ }^{45}$ G. Kasieczka, ${ }^{45}$ R. Klanner ${ }^{45}$ R. Kogler, ${ }^{45}$ T. Kramer, ${ }^{45}$ V. Kutzner, ${ }^{45}$ J. Lange, ${ }^{45}$ T. Lange, ${ }^{45}$ A. Lobanov, ${ }^{45}$ A. Malara, ${ }^{45}$ A. Nigamova, ${ }^{45}$ K. J. Pena Rodriguez, ${ }^{45}$ O. Rieger, ${ }^{45}$ J. Schindler, ${ }^{45}$ P. Schleper, ${ }^{45}$ M. Schröder, ${ }^{45}$ J. Schwandt, ${ }^{45}$ J. Sonneveld, ${ }^{45}$ H. Stadie, ${ }^{45}$ G. Steinbrück, ${ }^{45}$ A. Tews, ${ }^{45}$ I. Zoi, ${ }^{45}$ J. Bechtel, ${ }^{46}$ S. Brommer, ${ }^{46}$ E. Butz, ${ }^{46}$ R. Caspart, ${ }^{46}$ T. Chwalek, ${ }^{46}$ W. De Boer, ${ }^{46, a}$ A. Dierlamm, ${ }^{46}$ A. Droll, ${ }^{46}$ K. El Morabit, ${ }^{46}$ N. Faltermann, ${ }^{46}$ M. Giffels, ${ }^{46}$ J. o. Gosewisch, ${ }^{46}$ A. Gottmann, ${ }^{46}$ F. Hartmann, ${ }^{46, u}$ C. Heidecker, ${ }^{46}$ U. Husemann, ${ }^{46}$ P. Keicher, ${ }^{46}$ R. Koppenhöfer, ${ }^{46}$ S. Maier, ${ }^{46}$ M. Metzler, ${ }^{46}$ S. Mitra, ${ }^{46}$ Th. Müller, ${ }^{46}$ M. Neukum, ${ }^{46}$ A. Nürnberg, ${ }^{46}$ G. Quast, ${ }^{46}$ K. Rabbertz ${ }^{46}$ J. Rauser, ${ }^{46}$ D. Savoiu, ${ }^{46}$ M. Schnepf,${ }^{46}$ D. Seith, ${ }^{46}$ I. Shvetsov,${ }^{46}$ H. J. Simonis, ${ }^{46}$ R. Ulrich, ${ }^{46}$ J. Van Der Linden, ${ }^{46}$ R. F. Von Cube, ${ }^{46}$ M. Wassmer, ${ }^{46}$ M. Weber, ${ }^{46}$ S. Wieland, ${ }^{46}$ R. Wolf, ${ }^{46}$ S. Wozniewski, ${ }^{46}$ S. Wunsch, ${ }^{46}$ G. Anagnostou, ${ }^{47}$ G. Daskalakis ${ }^{47}$ T. Geralis, ${ }^{47}$ A. Kyriakis, ${ }^{47}$ D. Loukas, ${ }^{47}$ A. Stakia,${ }^{47}$ M. Diamantopoulou, ${ }^{48}$ D. Karasavvas, ${ }^{48}$ G. Karathanasis,${ }^{48}$ P. Kontaxakis, ${ }^{48}$ C. K. Koraka ${ }^{48}$ A. Manousakis-Katsikakis, ${ }^{48}$ A. Panagiotou, ${ }^{48}$ I. Papavergou, ${ }^{48}$ N. Saoulidou, ${ }^{48}$ K. Theofilatos, ${ }^{48}$ E. Tziaferi, ${ }^{48}$ K. Vellidis, ${ }^{48}$ E. Vourliotis, ${ }^{48}$ G. Bakas, ${ }^{49}$ K. Kousouris, ${ }^{49}$ I. Papakrivopoulos, ${ }^{49}$ G. Tsipolitis, ${ }^{49}$ A. Zacharopoulou, ${ }^{49}$ K. Adamidis, ${ }^{50}$ I. Bestintzanos, ${ }^{50}$ I. Evangelou, ${ }^{50}$ C. Foudas, ${ }^{50}$ P. Gianneios, ${ }^{50}$ P. Katsoulis,${ }^{50}$ P. Kokkas, ${ }^{50}$ N. Manthos,${ }^{50}$ I. Papadopoulos, ${ }^{50}$ J. Strologas,${ }^{50}$ M. Csanad, ${ }^{51}$ K. Farkas, ${ }^{51}$ M. M. A. Gadallah, ${ }^{51, \text { aa }}$ S. Lökös, ${ }^{51, b b}$ P. Major, ${ }^{51}$ K. Mandal, ${ }^{51}$ A. Mehta, ${ }^{51}$ G. Pasztor, ${ }^{51}$ A. J. Rádl, ${ }^{51}$ O. Surányi, ${ }^{51}$ G. I. Veres, ${ }^{51}$ M. Bartók, ${ }^{52, c c}$ G. Bencze, ${ }^{52}$ C. Hajdu,${ }^{52}$ D. Horvath, ${ }^{52, d d}$ F. Sikler ${ }^{52}$ V. Veszpremi, ${ }^{52}$ G. Vesztergombi, ${ }^{52, a, e e}$ S. Czellar, ${ }^{53}$ J. Karancsi, ${ }^{53, c c}$ J. Molnar, ${ }^{53}$ Z. Szillasi, ${ }^{53}$ D. Teyssier, ${ }^{53}$ P. Raics, ${ }^{54}$ Z. L. Trocsanyi, ${ }^{54, e e}$ B. Ujvari, ${ }^{54}$ 
T. Csorgo, ${ }^{55, f f}$ F. Nemes, ${ }^{55, f f}$ T. Novak, ${ }^{55}$ J. R. Komaragiri, ${ }^{56}$ D. Kumar,${ }^{56}$ L. Panwar, ${ }^{56}$ P. C. Tiwari, ${ }^{56}$ S. Bahinipati,,${ }^{57, g g}$ C. Kar, ${ }^{57}$ P. Mal, ${ }^{57}$ T. Mishra, ${ }^{57}$ V. K. Muraleedharan Nair Bindhu, ${ }^{57, \text { hh }}$ A. Nayak, ${ }^{57, \text { hh }}$ P. Saha, ${ }^{57}$ N. Sur, ${ }^{57}$ S. K. Swain, ${ }^{57}$ D. Vats ${ }^{57, \text { hh }}$ S. Bansal ${ }^{58}$ S. B. Beri, ${ }^{58}$ V. Bhatnagar, ${ }^{58}$ G. Chaudhary ${ }^{58}$ S. Chauhan, ${ }^{58}$ N. Dhingra, ${ }^{58, i i}$ R. Gupta, ${ }^{58}$ A. Kaur,${ }^{58}$ M. Kaur, ${ }^{58}$ S. Kaur, ${ }^{58}$ P. Kumari, ${ }^{58}$ M. Meena, ${ }^{58}$ K. Sandeep,${ }^{58}$ J. B. Singh ${ }^{58}$ A. K. Virdi, ${ }^{58}$ A. Ahmed, ${ }^{59}$ A. Bhardwaj, ${ }^{59}$ B. C. Choudhary, ${ }^{59}$ M. Gola,${ }^{59}$ S. Keshri, ${ }^{59}$ A. Kumar, ${ }^{59}$ M. Naimuddin, ${ }^{59}$ P. Priyanka ${ }^{59}$ K. Ranjan, ${ }^{59}$ A. Shah, ${ }^{59}$ M. Bharti, ${ }^{60, j \mathrm{ji}}$ R. Bhattacharya, ${ }^{60} \mathrm{~S}$. Bhattacharya, ${ }^{60}$ D. Bhowmik, ${ }^{60} \mathrm{~S}$. Dutta, ${ }^{60} \mathrm{~S}$. Dutta, ${ }^{60}$ B. Gomber, ${ }^{60, k \mathrm{k}}$ M. Maity, ${ }^{60,11}$ P. Palit, ${ }^{60}$ P. K. Rout, ${ }^{60}$ G. Saha ${ }^{60}$ B. Sahu, ${ }^{60}$ S. Sarkar, ${ }^{60}$ M. Sharan, ${ }^{60}$ B. Singh, ${ }^{60, j j}$ S. Thakur, ${ }^{60, j j}$ P. K. Behera, ${ }^{61}$ S. C. Behera, ${ }^{61}$ P. Kalbhor, ${ }^{61}$ A. Muhammad ${ }^{61}$ R. Pradhan,${ }^{61}$ P. R. Pujahari, ${ }^{61}$ A. Sharma, ${ }^{61}$ A. K. Sikdar, ${ }^{61}$ D. Dutta, ${ }^{62}$ V. Jha, ${ }^{62}$ V. Kumar, ${ }^{62}$ D. K. Mishra, ${ }^{62}$ K. Naskar, ${ }^{62, m m}$ P. K. Netrakanti, ${ }^{62}$ L. M. Pant, ${ }^{62}$ P. Shukla, ${ }^{62}$ T. Aziz, ${ }^{63}$ S. Dugad, ${ }^{63}$ M. Kumar, ${ }^{63}$ S. Banerjee, ${ }^{64}$ R. Chudasama ${ }^{64}$ M. Guchait ${ }^{64}$ S. Karmakar, ${ }^{64}$ S. Kumar, ${ }^{64}$ G. Majumder, ${ }^{64}$ K. Mazumdar,${ }^{64}$ S. Mukherjee, ${ }^{64}$ K. Alpana, ${ }^{65}$ S. Dube,${ }^{65}$ B. Kansal, ${ }^{65}$ A. Laha, ${ }^{65}$ S. Pandey, ${ }^{65}$ A. Rane, ${ }^{65}$ A. Rastogi, ${ }^{65}$ S. Sharma ${ }^{65}$ H. Bakhshiansohi, ${ }^{66, n n}$ E. Khazaie, ${ }^{66}$ M. Zeinali, ${ }^{66,00}$ S. Chenarani, ${ }^{67, p p}$ S. M. Etesami, ${ }^{67}$ M. Khakzad, ${ }^{67}$ M. Mohammadi Najafabadi, ${ }^{67}$ M. Grunewald, ${ }^{68}$ M. Abbrescia,${ }^{69,69 b}$ R. Aly, ${ }^{69 a, 69 b, q 9}$ C. Aruta ${ }^{69 a, 69 b}$ A. Colaleo, ${ }^{69 a}$ D. Creanza,${ }^{69 a, 69 c}$ N. De Filippis, ${ }^{69 a, 69 c}$ M. De Palma,${ }^{69 a, 69 b}$ A. Di Florio, ${ }^{69 a, 69 b}$ A. Di Pilato, ${ }^{69 a, 69 b}$ W. Elmetenawee, ${ }^{69 a, 69 b}$ L. Fiore ${ }^{69 \mathrm{a}}$ A. Gelmi, ${ }^{69 a, 69 b}$ M. Gul,${ }^{69 a}$ G. Iaselli, ${ }^{69 a, 69 c}$ M. Ince ${ }^{69 a, 69 b}$ S. Lezki ${ }^{69 a, 69 b}$ G. Maggi, ${ }^{69 a, 69 c}$ M. Maggi, ${ }^{69 a}$ I. Margjeka, ${ }^{69 a, 69 b}$ V. Mastrapasqua, ${ }^{69 a, 69 b}$ J. A. Merlin, ${ }^{69 a}$ S. My, ${ }^{69 a, 69 b}$ S. Nuzzo, ${ }^{69 a, 69 b}$ A. Pellecchia, ${ }^{69 a, 69 b}$ A. Pompili, ${ }^{69 a, 69 b}$ G. Pugliese,${ }^{69 a, 69 c}$ D. Ramos, ${ }^{69 a}$ A. Ranieri, ${ }^{69 a}$ G. Selvaggi, ${ }^{69 a, 69 b}$ L. Silvestris, ${ }^{69 a}$ F. M. Simone,${ }^{69 a, 69 b}$ R. Venditti, ${ }^{69 a}$ P. Verwilligen, ${ }^{69 \mathrm{a}}$ G. Abbiendi, ${ }^{70 \mathrm{a}}$ C. Battilana, ${ }^{70 \mathrm{a}, 70 \mathrm{~b}}$ D. Bonacorsi,${ }^{70 \mathrm{a}, 70 \mathrm{~b}}$ L. Borgonovi, ${ }^{70 \mathrm{a}}$ L. Brigliadori, ${ }^{70 \mathrm{a}}$

R. Campanini, ${ }^{70 a, 70 b}$ P. Capiluppi ${ }^{70 a, 70 b}$ A. Castro, ${ }^{70 a, 70 b}$ F. R. Cavallo, ${ }^{70 a}$ M. Cuffiani, ${ }^{70 a, 70 b}$ G. M. Dallavalle, ${ }^{70 a}$ T. Diotalevi, ${ }^{70 a, 70 b}$ F. Fabbri, ${ }^{70 a}$ A. Fanfani, ${ }^{70,70 b}$ P. Giacomelli, ${ }^{70 a}$ L. Giommi, ${ }^{70 a}, 70 \mathrm{~b}$ C. Grandi, ${ }^{70 a}$ L. Guiducci, ${ }^{70 a, 70 b}$ S. Lo Meo, ${ }^{70 a, r r}$ L. Lunerti, ${ }^{70 a, 70 b}$ S. Marcellini, ${ }^{70 a}$ G. Masetti, ${ }^{70 a}$ F. L. Navarria, ${ }^{70 a, 70 b}$ A. Perrotta, ${ }^{70 a}$ F. Primavera, ${ }^{70 a, 70 b}$ A. M. Rossi, ${ }^{70 a, 70 b}$ T. Rovelli, ${ }^{70 a, 70 b}$ G. P. Siroli, ${ }^{70 a, 70 b}$ S. Albergo,${ }^{71 a, 71 b, s s}$ S. Costa,${ }^{71 a, 71 b, s s}$ A. Di Mattia, ${ }^{71 a}$ R. Potenza, ${ }^{71 a, 71 b}$ A. Tricomi,${ }^{71 a, 71 b, s s}$ C. Tuve, ${ }^{71 a, 71 b}$ G. Barbagli, ${ }^{72 a}$ A. Cassese, ${ }^{72 a}$ R. Ceccarelli, ${ }^{72 a, 72 b}$ V. Ciulli, ${ }^{72 a, 72 b}$ C. Civinini, ${ }^{72 a}$ R. D’Alessandro, ${ }^{72 a, 72 b}$ E. Focardi, ${ }^{72 a, 72 b}$ G. Latino, ${ }^{72 a, 72 b}$ P. Lenzi, ${ }^{72 a, 72 b}$ M. Lizzo, ${ }^{72 a, 72 b}$ M. Meschini, ${ }^{72 a}$ S. Paoletti, ${ }^{72 a}$ R. Seidita, ${ }^{72 a, 72 b}$ G. Sguazzoni, ${ }^{72 a}$ L. Viliani, ${ }^{72 a}$ L. Benussi ${ }^{73}$ S. Bianco,${ }^{73}$ D. Piccolo, ${ }^{73}$ M. Bozzo, ${ }^{74 a, 74 b}$ F. Ferro ${ }^{74 a}$ R. Mulargia ${ }^{74 a, 74 b}$ E. Robutti, ${ }^{74 a}$ S. Tosi, ${ }^{74 a, 74 b}$ A. Benaglia, ${ }^{75 a}$ G. Boldrini, ${ }^{75 a}$ F. Brivio, ${ }^{75 a, 75 b}$ F. Cetorelli, ${ }^{75 a, 75 b}$ F. De Guio, ${ }^{75 a, 75 b}$ M. E. Dinardo, ${ }^{75 a, 75 b}$ P. Dini, ${ }^{75 a}$ S. Gennai, ${ }^{75 a}$ A. Ghezzi, ${ }^{75 a, 75 b}$ P. Govoni, ${ }^{75 a, 75 b}$ L. Guzzi,${ }^{75 a, 75 b}$ M. T. Lucchini ${ }^{75 a, 75 b}$ M. Malberti ${ }^{75 a}$ S. Malvezzi, ${ }^{75 a}$ A. Massironi, ${ }^{75 a}$ D. Menasce ${ }^{75 a}$ L. Moroni, ${ }^{75 a}$ M. Paganoni, ${ }^{75 a, 75 b}$ D. Pedrini, ${ }^{75 a}$ B. S. Pinolini, ${ }^{75 a}$ S. Ragazzi,${ }^{75 a, 75 b}$ N. Redaelli, ${ }^{75 a}$ T. Tabarelli de Fatis, ${ }^{75 a, 75 b}$ D. Valsecchi, ${ }^{75 a, 75 b, u}$ D. Zuolo, ${ }^{75 a, 75 b}$ S. Buontempo, ${ }^{76 a}$ F. Carnevali, ${ }^{76 a, 76 b}$ N. Cavallo, ${ }^{76 a, 76 c}$ A. De Iorio, ${ }^{76 a, 76 b}$ F. Fabozzi, ${ }^{76 a, 76 c}$ A. O. M. Iorio, ${ }^{76 a, 76 b}$ L. Lista, ${ }^{76 a, 76 b}$ S. Meola, ${ }^{76 a, 76 d, u}$ P. Paolucci, ${ }^{76 a, u}$ B. Rossi, ${ }^{76 a}$ C. Sciacca, ${ }^{76 a, 76 b}$ P. Azzi, ${ }^{77 a}$ N. Bacchetta, ${ }^{77 a}$ D. Bisello, ${ }^{77 a, 77 b}$ P. Bortignon, ${ }^{77 a}$ A. Bragagnolo, ${ }^{77 a, 77 b}$ R. Carlin,${ }^{77 a, 77 b}$ P. Checchia, ${ }^{77 a}$ T. Dorigo, ${ }^{77 a}$ U. Dosselli, ${ }^{77 a}$ F. Gasparini, ${ }^{77 a, 77 b}$ U. Gasparini, ${ }^{77 a, 77 b}$ G. Grosso, ${ }^{77 a}$ S. Y. Hoh, ${ }^{77 a, 77 b}$ L. Layer, ${ }^{77 a, t t}$ E. Lusiani, ${ }^{77 a}$ M. Margoni, ${ }^{77 a, 77 b}$ A. T. Meneguzzo, ${ }^{77 a, 77 b}$ J. Pazzini, ${ }^{77 a, 77 b}$ M. Presilla, ${ }^{77 a, 77 b}$ P. Ronchese, ${ }^{77 a, 77 b}$ R. Rossin, ${ }^{77 a, 77 b}$ F. Simonetto, ${ }^{77 a, 77 b}$ G. Strong, ${ }^{77 a}$ M. Tosi, ${ }^{77 a, 77 b}$ H. Yarar, ${ }^{77,77 b}$ M. Zanetti, ${ }^{77 a, 77 b}$ P. Zotto, ${ }^{77 a, 77 b}$ A. Zucchetta, ${ }^{77 a, 77 b}$ G. Zumerle, ${ }^{77 a, 77 b}$ C. Aime ${ }^{78 a, 78 b}$ A. Braghieri, ${ }^{78 a}$ S. Calzaferri, ${ }^{78 a, 78 b}$ D. Fiorina, ${ }^{78 a, 78 b}$ P. Montagna, ${ }^{78 a, 78 b}$ S. P. Ratti, ${ }^{78 a, 78 b}$ V. Re, ${ }^{78 a}$ C. Riccardi, ${ }^{78 a, 78 b}$ P. Salvini, ${ }^{78 a}$ I. Vai, ${ }^{78 a}$ P. Vitulo, ${ }^{78 a, 78 b}$ P. Asenov, ${ }^{79 a, \text { uи }}$ G. M. Bilei ${ }^{79 a}$ D. Ciangottini, ${ }^{79 a, 79 b}$

L. Fanò ${ }^{79 a, 79 b}$ P. Lariccia ${ }^{79 a, 79 b}$ M. Magherini ${ }^{79 a, 79 b}$ G. Mantovani ${ }^{79 a, 79 b}$ V. Mariani, ${ }^{79 a, 79 b}$ M. Menichelli, ${ }^{79 a}$ F. Moscatelli, ${ }^{79 a \text {,u }}$ A. Piccinelli, ${ }^{79 a, 79 b}$ A. Rossi,${ }^{79 a, 79 b}$ A. Santocchia,${ }^{79 a, 79 b}$ D. Spiga, ${ }^{79 a}$ T. Tedeschi, ${ }^{79 a, 79 b}$ P. Azzurri, ${ }^{80 a}$ G. Bagliesi, ${ }^{80 \mathrm{a}}$ V. Bertacchi, ${ }^{80 a}, 80 \mathrm{c}$ L. Bianchini, ${ }^{80 \mathrm{a}}$ T. Boccali, ${ }^{80 \mathrm{a}}$ E. Bossini, ${ }^{80 \mathrm{a}, 80 \mathrm{~b}}$ R. Castaldi, ${ }^{80 \mathrm{a}}$ M. A. Ciocci, ${ }^{80,80 \mathrm{~b}}$ V. D’Amante, ${ }^{80 a, 80 \mathrm{~d}}$ R. Dell'Orso, ${ }^{80 \mathrm{a}}$ M. R. Di Domenico, ${ }^{80 \mathrm{a}, 80 \mathrm{~d}}$ S. Donato, ${ }^{80 \mathrm{a}}$ A. Giassi, ${ }^{80 \mathrm{a}}$ F. Ligabue, ${ }^{80 \mathrm{a}, 80 \mathrm{c}}$ E. Manca, ${ }^{80 \mathrm{a}, 80 \mathrm{c}}$ G. Mandorli, ${ }^{80 a, 80 c}$ A. Messineo, ${ }^{80 a, 80 b}$ F. Palla, ${ }^{80 a}$ S. Parolia, ${ }^{80 a, 80 b}$ G. Ramirez-Sanchez, ${ }^{80 a, 80 c}$ A. Rizzi, ${ }^{80 a, 80 b}$ G. Rolandi, ${ }^{80 a, 80 \mathrm{c}}$ S. Roy Chowdhury, ${ }^{80 a, 80 \mathrm{c}}$ A. Scribano, ${ }^{80 \mathrm{a}}$ N. Shafiei,${ }^{80 \mathrm{a}, 80 \mathrm{~b}}$ P. Spagnolo, ${ }^{80 \mathrm{a}}$ R. Tenchini, ${ }^{80 \mathrm{a}}$ G. Tonelli, ${ }^{80 \mathrm{a}, 80 \mathrm{~b}}$ N. Turini,${ }^{80 a, 80 d}$ A. Venturi, ${ }^{80 \mathrm{a}}$ P. G. Verdini, ${ }^{80 \mathrm{a}}$ P. Barria,${ }^{81 \mathrm{a}}$ M. Campana,${ }^{81 \mathrm{a}, 81 \mathrm{~b}}$ F. Cavallari, ${ }^{81 \mathrm{a}}$ D. Del Re,${ }^{81 \mathrm{a}, 81 \mathrm{~b}}$ E. Di Marco, ${ }^{81 \mathrm{a}}$ M. Diemoz, ${ }^{81 \mathrm{a}}$ E. Longo, ${ }^{81 \mathrm{a}, 81 \mathrm{~b}}$ P. Meridiani, ${ }^{81 \mathrm{a}}$ G. Organtini, ${ }^{81,81 \mathrm{~b}}$ F. Pandolfi, ${ }^{81 \mathrm{a}}$ R. Paramatti, ${ }^{81 \mathrm{a}, 81 \mathrm{~b}}$ C. Quaranta, ${ }^{81 a, 81 b}$ S. Rahatlou, ${ }^{81 a, 81 b}$ C. Rovelli, ${ }^{81 a}$ F. Santanastasio, ${ }^{81 a, 81 b}$ L. Soffi, ${ }^{81 a}$ R. Tramontano,${ }^{81 a, 81 b}$ N. Amapane, ${ }^{82 a, 82 b}$ R. Arcidiacono, ${ }^{82 a, 82 c}$ S. Argiro, ${ }^{82 a, 82 b}$ M. Arneodo, ${ }^{82 a, 82 c}$ N. Bartosik, ${ }^{82 a}$ R. Bellan, ${ }^{82 a, 82 b}$ A. Bellora, ${ }^{82 a, 82 b}$ J. Berenguer Antequera, ${ }^{82 a, 82 b}$ C. Biino, ${ }^{82 a}$ N. Cartiglia, ${ }^{82 a}$ S. Cometti, ${ }^{82 a}$ M. Costa, ${ }^{82 a, 82 b}$ R. Covarelli, ${ }^{82 a, 82 b}$ N. Demaria, ${ }^{82 a}$ B. Kiani, ${ }^{82 a, 82 b}$ F. Legger, ${ }^{82 a}$ C. Mariotti, ${ }^{82 a}$ S. Maselli, ${ }^{82 a}$ E. Migliore, ${ }^{82 a, 82 b}$ E. Monteil, ${ }^{82 a, 82 b}$ M. Monteno, ${ }^{82 a}$ 
M. M. Obertino, ${ }^{82 a, 82 b}$ G. Ortona, ${ }^{82 a}$ L. Pacher, ${ }^{82 a, 82 b}$ N. Pastrone, ${ }^{82 a}$ M. Pelliccioni, ${ }^{82 a}$ G. L. Pinna Angioni, ${ }^{82 a, 82 b}$ M. Ruspa, ${ }^{82 a, 82 c}$ K. Shchelina, ${ }^{82 a}$ F. Siviero, ${ }^{82 a, 82 b}$ V. Sola, ${ }^{82 a}$ A. Solano, ${ }^{82 a, 82 b}$ D. Soldi, ${ }^{82 a, 82 b}$ A. Staiano, ${ }^{82 a}$ M. Tornago, ${ }^{82 a, 82 b}$ D. Trocino, ${ }^{82 a}$ A. Vagnerini, ${ }^{82 a, 82 b}$ S. Belforte, ${ }^{83 a}$ V. Candelise, ${ }^{83 a, 83 b}$ M. Casarsa ${ }^{83 a}$ F. Cossutti, ${ }^{83 a}$ A. Da Rold, ${ }^{83 a, 83 b}$ G. Della Ricca, ${ }^{83 a, 83 b}$ G. Sorrentino, ${ }^{83 a, 83 b}$ F. Vazzoler, ${ }^{83 a, 83 b}$ S. Dogra, ${ }^{84}$ C. Huh, ${ }^{84}$ B. Kim, ${ }^{84}$ D. H. Kim, ${ }^{84}$ G. N. Kim, ${ }^{84}$ J. Kim, ${ }^{84}$ J. Lee, ${ }^{84}$ S. W. Lee, ${ }^{84}$ C. S. Moon, ${ }^{84}$ Y. D. Oh, ${ }^{84}$ S. I. Pak, ${ }^{84}$ B. C. Radburn-Smith, ${ }^{84}$ S. Sekmen, ${ }^{84}$ Y. C. Yang, ${ }^{84}$ H. Kim, ${ }^{85}$ D. H. Moon, ${ }^{85}$ B. Francois, ${ }^{86}$ T. J. Kim, ${ }^{86}$ J. Park, ${ }^{86}$ S. Cho, ${ }^{87}$ S. Choi, ${ }^{87}$ Y. Go, ${ }^{87}$ B. Hong, ${ }^{87}$ K. Lee, ${ }^{87}$ K. S. Lee, ${ }^{87}$ J. Lim, ${ }^{87}$ J. Park, ${ }^{87}$ S. K. Park, ${ }^{87}$ J. Yoo, ${ }^{87}$ J. Goh, ${ }^{88}$ A. Gurtu, ${ }^{88}$ H. S. Kim, ${ }^{89}$ Y. Kim, ${ }^{89}$ J. Almond, ${ }^{90}$ J. H. Bhyun, ${ }^{90}$ J. Choi, ${ }^{90}$ S. Jeon, ${ }^{90}$ J. Kim, ${ }^{90}$ J. S. Kim, ${ }^{90}$ S. Ko, ${ }^{90}$ H. Kwon, ${ }^{90}$ H. Lee, ${ }^{90}$ S. Lee, ${ }^{90}$ B. H. Oh, ${ }^{90}$ M. Oh, ${ }^{90}$ S. B. Oh, ${ }^{90}$ H. Seo, ${ }^{90}$ U. K. Yang, ${ }^{90}$ I. Yoon, ${ }^{90}$ W. Jang, ${ }^{91}$ D. Y. Kang, ${ }^{91}$ Y. Kang, ${ }^{91}$ S. Kim, ${ }^{91}$ B. Ko, ${ }^{91}$ J. S. H. Lee, ${ }^{91}$ Y. Lee, ${ }^{91}$ I. C. Park, ${ }^{91}$ Y. Roh, ${ }^{91}$ M. S. Ryu, ${ }^{91}$ D. Song, ${ }^{91}$ I. J. Watson, ${ }^{91}$ S. Yang, ${ }^{91}$ S. Ha, ${ }^{92}$ H. D. Yoo, ${ }^{92}$ M. Choi, ${ }^{93}$ H. Lee, ${ }^{93}$ Y. Lee, ${ }^{93}$ I. Yu, ${ }^{93}$ T. Beyrouthy, ${ }^{94}$ Y. Maghrbi, ${ }^{94}$ T. Torims, ${ }^{95}$ V. Veckalns, ${ }^{95, v v}$ M. Ambrozas, ${ }^{96}$ A. Carvalho Antunes De Oliveira, ${ }^{96}$ A. Juodagalvis, ${ }^{96}$ A. Rinkevicius, ${ }^{96}$ G. Tamulaitis, ${ }^{96}$ N. Bin Norjoharuddeen, ${ }^{97}$ W. A. T. Wan Abdullah, ${ }^{97}$ M. N. Yusli, ${ }^{97}$ Z. Zolkapli, ${ }^{97}$ J. F. Benitez, ${ }^{98}$ A. Castaneda Hernandez, ${ }^{98}$ M. León Coello, ${ }^{98}$ J. A. Murillo Quijada, ${ }^{98}$ A. Sehrawat, ${ }^{98}$

L. Valencia Palomo, ${ }^{98}$ G. Ayala, ${ }^{99}$ H. Castilla-Valdez, ${ }^{99}$ E. De La Cruz-Burelo, ${ }^{99}$ I. Heredia-De La Cruz, ${ }^{99, w w}$

R. Lopez-Fernandez, ${ }^{99}$ C. A. Mondragon Herrera, ${ }^{99}$ D. A. Perez Navarro, ${ }^{99}$ A. Sanchez-Hernandez, ${ }^{99}$ S. Carrillo Moreno, ${ }^{100}$

C. Oropeza Barrera, ${ }^{100}$ F. Vazquez Valencia, ${ }^{100}$ I. Pedraza, ${ }^{101}$ H. A. Salazar Ibarguen, ${ }^{101}$ C. Uribe Estrada, ${ }^{101}$

J. Mijuskovic, ${ }^{102, \mathrm{xx}}$ N. Raicevic, ${ }^{102}$ D. Krofcheck, ${ }^{103}$ P. H. Butler, ${ }^{104}$ A. Ahmad, ${ }^{105}$ M. I. Asghar, ${ }^{105}$ A. Awais, ${ }^{105}$ M. I. M. Awan, ${ }^{105}$ H. R. Hoorani, ${ }^{105}$ W. A. Khan, ${ }^{105}$ M. A. Shah, ${ }^{105}$ M. Shoaib,${ }^{105}$ M. Waqas, ${ }^{105}$ V. Avati, ${ }^{106}$ L. Grzanka, ${ }^{106}$ M. Malawski, ${ }^{106}$ H. Bialkowska, ${ }^{107}$ M. Bluj, ${ }^{107}$ B. Boimska, ${ }^{107}$ M. Górski, ${ }^{107}$ M. Kazana, ${ }^{107}$ M. Szleper, ${ }^{107}$ P. Zalewski, ${ }^{107}$ K. Bunkowski, ${ }^{108}$ K. Doroba, ${ }^{108}$ A. Kalinowski, ${ }^{108}$ M. Konecki, ${ }^{108}$ J. Krolikowski, ${ }^{108}$ M. Walczak, ${ }^{108}$ M. Araujo, ${ }^{109}$ P. Bargassa, ${ }^{109}$ D. Bastos, ${ }^{109}$ A. Boletti, ${ }^{109}$ P. Faccioli, ${ }^{109}$ M. Gallinaro, ${ }^{109}$ J. Hollar, ${ }^{109}$ N. Leonardo, ${ }^{109}$ T. Niknejad, ${ }^{109}$ M. Pisano, ${ }^{109}$ J. Seixas, ${ }^{109}$ O. Toldaiev, ${ }^{109}$ J. Varela, ${ }^{109}$ S. Afanasiev, ${ }^{110}$ D. Budkouski, ${ }^{110}$ I. Golutvin, ${ }^{110}$ I. Gorbunov, ${ }^{110}$ V. Karjavine, ${ }^{110}$ V. Korenkov, ${ }^{110}$ A. Lanev, ${ }^{110}$ A. Malakhov, ${ }^{110}$ V. Matveev, ${ }^{110, y y, z z}$ V. Palichik, ${ }^{110}$ V. Perelygin, ${ }^{110}$ M. Savina, ${ }^{110}$ D. Seitova, ${ }^{110}$ V. Shalaev, ${ }^{110}$ S. Shmatov, ${ }^{110}$ S. Shulha, ${ }^{110}$ V. Smirnov, ${ }^{110}$ O. Teryaev, ${ }^{110}$ N. Voytishin, ${ }^{110}$ B. S. Yuldashev, ${ }^{110, \text { aaa }}$ A. Zarubin, ${ }^{110}$ I. Zhizhin, ${ }^{110}$ G. Gavrilov, ${ }^{111}$ V. Golovtcov, ${ }^{111}$ Y. Ivanov, ${ }^{111}$ V. Kim, ${ }^{111, b b b}$ E. Kuznetsova, ${ }^{11, \text { ccc }}$ V. Murzin, ${ }^{111}$ V. Oreshkin, ${ }^{111}$ I. Smirnov, ${ }^{111}$ D. Sosnov, ${ }^{111}$ V. Sulimov, ${ }^{111}$ L. Uvarov, ${ }^{111}$ S. Volkov, ${ }^{111}$ A. Vorobyev, ${ }^{111}$ Yu. Andreev, ${ }^{112}$ A. Dermenev, ${ }^{112}$ S. Gninenko, ${ }^{112}$ N. Golubev, ${ }^{112}$ A. Karneyeu, ${ }^{112}$ D. Kirpichnikov, ${ }^{112}$ M. Kirsanov, ${ }^{112}$ N. Krasnikov, ${ }^{112}$ A. Pashenkov, ${ }^{112}$ G. Pivovarov, ${ }^{112}$ A. Toropin, ${ }^{112}$ V. Epshteyn, ${ }^{113}$ V. Gavrilov, ${ }^{113}$ N. Lychkovskaya, ${ }^{113}$ A. Nikitenko, ${ }^{113, \text { ddd }}$ V. Popov, ${ }^{113}$ A. Stepennov, ${ }^{113}$ M. Toms, ${ }^{113}$ E. Vlasov, ${ }^{113}$ A. Zhokin, ${ }^{113}$ T. Aushev, ${ }^{114}$ O. Bychkova, ${ }^{115}$ R. Chistov, ${ }^{115, \text { eee }}$ M. Danilov, ${ }^{115, \text { eee }}$ A. Oskin, ${ }^{115}$ P. Parygin, ${ }^{115}$ S. Polikarpov, ${ }^{115, \text { eee }}$ V. Andreev, ${ }^{116}$ M. Azarkin, ${ }^{116}$ I. Dremin, ${ }^{116}$ M. Kirakosyan, ${ }^{116}$ A. Terkulov, ${ }^{116}$ A. Belyaev, ${ }^{117}$ E. Boos, ${ }^{117}$ V. Bunichev, ${ }^{117}$ M. Dubinin, ${ }^{117, f f f}$ L. Dudko, ${ }^{117}$ A. Ershov, ${ }^{117}$ V. Klyukhin, ${ }^{117}$ O. Kodolova, ${ }^{117}$ I. Lokhtin, ${ }^{117}$ S. Obraztsov, ${ }^{117}$ M. Perfilov, ${ }^{117}$ S. Petrushanko, ${ }^{117}$ V. Savrin, ${ }^{117}$ V. Blinov, ${ }^{118, g g g}$ T. Dimova, ${ }^{118, \text { ggg }}$ L. Kardapoltsev, ${ }^{118, g g g}$ A. Kozyrev, ${ }^{118, g g g}$ I. Ovtin, ${ }^{118, g g g}$ Y. Skovpen, ${ }^{118, g g g}$ I. Azhgirey, ${ }^{119}$ I. Bayshev, ${ }^{119}$ D. Elumakhov, ${ }^{119}$ V. Kachanov, ${ }^{119}$ D. Konstantinov, ${ }^{119}$ P. Mandrik, ${ }^{119}$ V. Petrov, ${ }^{119}$ R. Ryutin, ${ }^{119}$ S. Slabospitskii, ${ }^{119}$ A. Sobol, ${ }^{119}$ S. Troshin, ${ }^{119}$ N. Tyurin, ${ }^{119}$ A. Uzunian, ${ }^{119}$ A. Volkov, ${ }^{119}$ A. Babaev, ${ }^{120}$ V. Okhotnikov, ${ }^{120}$ V. Borshch, ${ }^{121}$ V. Ivanchenko, ${ }^{121}$ E. Tcherniaev, ${ }^{121}$ P. Adzic, ${ }^{122, \text { hhh }}$ M. Dordevic, ${ }^{122}$ P. Milenovic, ${ }^{122}$ J. Milosevic, ${ }^{122}$ M. Aguilar-Benitez, ${ }^{123}$ J. Alcaraz Maestre, ${ }^{123}$ A. Álvarez Fernández, ${ }^{123}$ I. Bachiller, $^{123}$ M. Barrio Luna, ${ }^{123}$ C. F. Bedoya, ${ }^{123}$ C. A. Carrillo Montoya, ${ }^{123}$ M. Cepeda, ${ }^{123}$ M. Cerrada, ${ }^{123}$ N. Colino, ${ }^{123}$

B. De La Cruz, ${ }^{123}$ A. Delgado Peris, ${ }^{123}$ J. P. Fernández Ramos, ${ }^{123}$ J. Flix, ${ }^{123}$ M. C. Fouz, ${ }^{123}$ O. Gonzalez Lopez, ${ }^{123}$

S. Goy Lopez, ${ }^{123}$ J. M. Hernandez, ${ }^{123}$ M. I. Josa, ${ }^{123}$ J. León Holgado, ${ }^{123}$ D. Moran, ${ }^{123}$ Á Navarro Tobar, ${ }^{123}$

C. Perez Dengra, ${ }^{123}$ A. Pérez-Calero Yzquierdo, ${ }^{123}$ J. Puerta Pelayo, ${ }^{123}$ I. Redondo, ${ }^{123}$ L. Romero, ${ }^{123}$ S. Sánchez Navas, ${ }^{123}$ L. Urda Gómez, ${ }^{123}$ C. Willmott, ${ }^{123}$ J. F. de Trocóniz, ${ }^{124}$ R. Reyes-Almanza, ${ }^{124}$ B. Alvarez Gonzalez, ${ }^{125}$ J. Cuevas, ${ }^{125}$ C. Erice, ${ }^{125}$ J. Fernandez Menendez, ${ }^{125}$ S. Folgueras, ${ }^{125}$ I. Gonzalez Caballero, ${ }^{125}$ J. R. González Fernández, ${ }^{125}$ E. Palencia Cortezon, ${ }^{125}$ C. Ramón Álvarez, ${ }^{125}$ V. Rodríguez Bouza, ${ }^{125}$ A. Soto Rodríguez, ${ }^{125}$ A. Trapote, ${ }^{125}$ N. Trevisani, ${ }^{125}$ C. Vico Villalba, ${ }^{125}$ J. A. Brochero Cifuentes, ${ }^{126}$ I. J. Cabrillo, ${ }^{126}$ A. Calderon, ${ }^{126}$ J. Duarte Campderros, ${ }^{126}$ M. Fernandez, ${ }^{126}$ C. Fernandez Madrazo, ${ }^{126}$ P. J. Fernández Manteca, ${ }^{126}$ A. García Alonso, ${ }^{126}$ G. Gomez, ${ }^{126}$ C. Martinez Rivero, ${ }^{126}$

P. Martinez Ruiz del Arbol, ${ }^{126}$ F. Matorras, ${ }^{126}$ Pablo Matorras-Cuevas, ${ }^{126}$ J. Piedra Gomez, ${ }^{126}$ C. Prieels, ${ }^{126}$ T. Rodrigo, ${ }^{126}$ A. Ruiz-Jimeno, ${ }^{126}$ L. Scodellaro, ${ }^{126}$ I. Vila, ${ }^{126}$ J. M. Vizan Garcia, ${ }^{126}$ M. K. Jayananda, ${ }^{127}$ B. Kailasapathy, ${ }^{127, \text { iii }}$ D. U. J. Sonnadara, ${ }^{127}$ D. D. C. Wickramarathna, ${ }^{127}$ W. G. D. Dharmaratna, ${ }^{128}$ K. Liyanage, ${ }^{128}$ N. Perera, ${ }^{128}$ 
N. Wickramage, ${ }^{128}$ T. K. Aarrestad, ${ }^{129}$ D. Abbaneo, ${ }^{129}$ J. Alimena, ${ }^{129}$ E. Auffray, ${ }^{129}$ G. Auzinger, ${ }^{129}$ J. Baechler, ${ }^{129}$ P. Baillon, ${ }^{129, a}$ D. Barney, ${ }^{129}$ J. Bendavid, ${ }^{129}$ M. Bianco, ${ }^{129}$ A. Bocci, ${ }^{129}$ T. Camporesi, ${ }^{129}$ M. Capeans Garrido, ${ }^{129}$ G. Cerminara, ${ }^{129}$ S. S. Chhibra, ${ }^{129}$ M. Cipriani, ${ }^{129}$ L. Cristella, ${ }^{129}$ D. d'Enterria, ${ }^{129}$ A. Dabrowski, ${ }^{129}$ A. David, ${ }^{129}$ A. De Roeck, ${ }^{129}$ M. M. Defranchis, ${ }^{129}$ M. Deile, ${ }^{129}$ M. Dobson,${ }^{129}$ M. Dünser, ${ }^{129}$ N. Dupont, ${ }^{129}$ A. Elliott-Peisert, ${ }^{129}$ N. Emriskova, ${ }^{129}$ F. Fallavollita, ${ }^{129, j \mathrm{jj}}$ D. Fasanella, ${ }^{129}$ A. Florent, ${ }^{129}$ G. Franzoni, ${ }^{129}$ W. Funk, ${ }^{129}$ S. Giani, ${ }^{129}$ D. Gigi, ${ }^{129}$ K. Gill, ${ }^{129}$ F. Glege, ${ }^{129}$ L. Gouskos, ${ }^{129}$ M. Haranko, ${ }^{129}$ J. Hegeman, ${ }^{129}$ V. Innocente, ${ }^{129}$ T. James, ${ }^{129}$ P. Janot, ${ }^{129}$ J. Kaspar, ${ }^{129}$ J. Kieseler, ${ }^{129}$ M. Komm, ${ }^{129}$ N. Kratochwil, ${ }^{129}$ C. Lange, ${ }^{129}$ S. Laurila, ${ }^{129}$ P. Lecoq, ${ }^{129}$ A. Lintuluoto, ${ }^{129}$ K. Long, ${ }^{129}$ C. Lourenço, ${ }^{129}$ B. Maier, ${ }^{129}$ L. Malgeri, ${ }^{129}$ S. Mallios, ${ }^{129}$ M. Mannelli, ${ }^{129}$ A. C. Marini, ${ }^{129}$ F. Meijers, ${ }^{129}$ S. Mersi, ${ }^{129}$ E. Meschi, ${ }^{129}$ F. Moortgat, ${ }^{129}$ M. Mulders, ${ }^{129}$ S. Orfanelli, ${ }^{129}$ L. Orsini, ${ }^{129}$ F. Pantaleo, ${ }^{129}$ L. Pape, ${ }^{129}$ E. Perez, ${ }^{129}$ M. Peruzzi, ${ }^{129}$ A. Petrilli, ${ }^{129}$ G. Petrucciani, ${ }^{129}$ A. Pfeiffer, ${ }^{129}$ M. Pierini, ${ }^{129}$ D. Piparo, ${ }^{129}$ M. Pitt, ${ }^{129}$ H. Qu, ${ }^{129}$ T. Quast, ${ }^{129}$ D. Rabady, ${ }^{129}$ A. Racz, ${ }^{129}$ G. Reales Gutiérrez, ${ }^{129}$ M. Rieger, ${ }^{129}$ M. Rovere, ${ }^{129}$ H. Sakulin, ${ }^{129}$ J. Salfeld-Nebgen, ${ }^{129}$

S. Scarfi, ${ }^{129}$ C. Schäfer, ${ }^{129}$ C. Schwick, ${ }^{129}$ M. Selvaggi, ${ }^{129}$ A. Sharma, ${ }^{129}$ P. Silva, ${ }^{129}$ W. Snoeys, ${ }^{129}$ P. Sphicas, ${ }^{129, k k k}$ S. Summers, ${ }^{129}$ K. Tatar, ${ }^{129}$ V. R. Tavolaro, ${ }^{129}$ D. Treille, ${ }^{129}$ P. Tropea, ${ }^{129}$ A. Tsirou, ${ }^{129}$ G. P. Van Onsem, ${ }^{129}$ J. Wanczyk, ${ }^{129,111}$ K. A. Wozniak, ${ }^{129}$ W. D. Zeuner, ${ }^{129}$ L. Caminada, ${ }^{130, m m m}$ A. Ebrahimi, ${ }^{130}$ W. Erdmann, ${ }^{130}$ R. Horisberger, ${ }^{130}$ Q. Ingram, ${ }^{130}$ H. C. Kaestli, ${ }^{130}$ D. Kotlinski, ${ }^{130}$ U. Langenegger, ${ }^{130}$ M. Missiroli, ${ }^{130}$ L. Noehte, ${ }^{130}$ T. Rohe, ${ }^{130}$ K. Androsov, ${ }^{131,111}$ M. Backhaus ${ }^{131}$ P. Berger,${ }^{131}$ A. Calandri, ${ }^{131}$ N. Chernyavskaya,${ }^{131}$ A. De Cosa,${ }^{131}$ G. Dissertori, ${ }^{131}$ M. Dittmar, ${ }^{131}$ M. Donegà, ${ }^{131}$ C. Dorfer, ${ }^{131}$ F. Eble, ${ }^{131}$ K. Gedia ${ }^{131}$ F. Glessgen, ${ }^{131}$ T. A. Gómez Espinosa, ${ }^{131}$ C. Grab,${ }^{131}$ D. Hits, ${ }^{131}$ W. Lustermann, ${ }^{131}$ A.-M. Lyon, ${ }^{131}$ R. A. Manzoni, ${ }^{131}$ L. Marchese, ${ }^{131}$ C. Martin Perez, ${ }^{131}$ M. T. Meinhard,${ }^{131}$

F. Nessi-Tedaldi, ${ }^{131}$ J. Niedziela, ${ }^{131}$ F. Pauss, ${ }^{131}$ V. Perovic, ${ }^{131}$ S. Pigazzini,${ }^{131}$ M. G. Ratti, ${ }^{131}$ M. Reichmann, ${ }^{131}$ C. Reissel, ${ }^{131}$ T. Reitenspiess, ${ }^{131}$ B. Ristic, ${ }^{131}$ D. Ruini, ${ }^{131}$ D. A. Sanz Becerra, ${ }^{131}$ V. Stampf,,${ }^{131}$ J. Steggemann, ${ }^{131,111}$ R. Wallny, ${ }^{131}$ D. H. Zhu, ${ }^{131}$ C. Amsler, ${ }^{132, n n n}$ P. Bärtschi, ${ }^{132}$ C. Botta, ${ }^{132}$ D. Brzhechko, ${ }^{132}$ M. F. Canelli, ${ }^{132}$ K. Cormier, ${ }^{132}$ A. De Wit, ${ }^{132}$ R. Del Burgo, ${ }^{132}$ J. K. Heikkilä, ${ }^{132}$ M. Huwiler, ${ }^{132}$ W. Jin, ${ }^{132}$ A. Jofrehei ${ }^{132}$ B. Kilminster, ${ }^{132}$ S. Leontsinis, ${ }^{132}$

S. P. Liechti, ${ }^{132}$ A. Macchiolo, ${ }^{132}$ P. Meiring, ${ }^{132}$ V. M. Mikuni, ${ }^{132}$ U. Molinatti ${ }^{132}$ I. Neutelings, ${ }^{132}$ A. Reimers, ${ }^{132}$ P. Robmann, ${ }^{132}$ S. Sanchez Cruz, ${ }^{132}$ K. Schweiger, ${ }^{132}$ Y. Takahashi, ${ }^{132}$ C. Adloff, ${ }^{133,000}$ C. M. Kuo, ${ }^{133}$ W. Lin,${ }^{133}$ A. Roy, ${ }^{133}$ T. Sarkar, ${ }^{133,11}$ S. S. Yu, ${ }^{133}$ L. Ceard,${ }^{134}$ Y. Chao, ${ }^{134}$ K. F. Chen, ${ }^{134}$ P. H. Chen, ${ }^{134}$ W.-S. Hou, ${ }^{134}$ Y. y. Li ${ }^{134}$ R.-S. Lu, ${ }^{134}$ E. Paganis, ${ }^{134}$ A. Psallidas, ${ }^{134}$ A. Steen, ${ }^{134}$ H. y. Wu, ${ }^{134}$ E. Yazgan, ${ }^{134}$ P. r. Yu, ${ }^{134}$ B. Asavapibhop, ${ }^{135}$

C. Asawatangtrakuldee, ${ }^{135}$ N. Srimanobhas, ${ }^{135}$ F. Boran, ${ }^{136}$ S. Damarseckin, ${ }^{136, p p p}$ Z. S. Demiroglu, ${ }^{136}$ F. Dolek, ${ }^{136}$

I. Dumanoglu, ${ }^{136, \text { qqq }}$ E. Eskut, ${ }^{136}$ Y. Guler, ${ }^{136, \text { rrr }}$ E. Gurpinar Guler,${ }^{136, \text { rrr }}$ I. Hos, ${ }^{136, \text { sss }}$ C. Isik, ${ }^{136}$ O. Kara, ${ }^{136}$

A. Kayis Topaksu, ${ }^{136}$ U. Kiminsu, ${ }^{136}$ G. Onengut, ${ }^{136}$ K. Ozdemir, ${ }^{136, t t t}$ A. Polatoz, ${ }^{136}$ A. E. Simsek, ${ }^{136}$ B. Tali, ${ }^{136, \text { uuu }}$ U. G. Tok, ${ }^{136}$ S. Turkcapar, ${ }^{136}$ I. S. Zorbakir, ${ }^{136}$ C. Zorbilmez, ${ }^{136}$ B. Isildak, ${ }^{137, \mathrm{vvv}}$ G. Karapinar, ${ }^{137, \mathrm{www}}$ K. Ocalan, ${ }^{137, \mathrm{xxx}}$ M. Yalvac, ${ }^{137, y y y}$ B. Akgun, ${ }^{138}$ I. O. Atakisi, ${ }^{138}$ E. Gülmez, ${ }^{138}$ M. Kaya, ${ }^{138, z z z}$ O. Kaya,${ }^{138, \text { aaaa }}$ Ö. Özçelik, ${ }^{138}$ S. Tekten, ${ }^{138, \text { bbbb }}$

E. A. Yetkin, ${ }^{138, \text { cccc }}$ A. Cakir, ${ }^{139}$ K. Cankocak, ${ }^{139, \text { qq9 }}$ Y. Komurcu, ${ }^{139}$ S. Sen, ${ }^{139, \text { dddd }}$ S. Cerci, ${ }^{140, \text { uuu }}$ B. Kaynak, ${ }^{140}$

S. Ozkorucuklu, ${ }^{140}$ D. Sunar Cerci, ${ }^{140, \text { uuu }}$ B. Grynyov, ${ }^{141}$ L. Levchuk, ${ }^{142}$ D. Anthony, ${ }^{143}$ E. Bhal, ${ }^{143}$ S. Bologna, ${ }^{143}$ J. J. Brooke, ${ }^{143}$ A. Bundock, ${ }^{143}$ E. Clement, ${ }^{143}$ D. Cussans, ${ }^{143}$ H. Flacher, ${ }^{143}$ J. Goldstein, ${ }^{143}$ G. P. Heath, ${ }^{143}$ H. F. Heath, ${ }^{143}$ L. Kreczko, ${ }^{143}$ B. Krikler, ${ }^{143}$ S. Paramesvaran, ${ }^{143}$ S. Seif El Nasr-Storey, ${ }^{143}$ V. J. Smith, ${ }^{143}$ N. Stylianou, ${ }^{143, \text { eeee }}$

K. Walkingshaw Pass, ${ }^{143}$ R. White, ${ }^{143}$ K. W. Bell, ${ }^{144}$ A. Belyaev, ${ }^{144, f f f f}$ C. Brew, ${ }^{144}$ R. M. Brown, ${ }^{144}$ D. J. A. Cockerill, ${ }^{144}$ C. Cooke,${ }^{144}$ K. V. Ellis, ${ }^{144}$ K. Harder, ${ }^{144}$ S. Harper, ${ }^{144}$ M. 1 . Holmberg, ${ }^{144, g g g g}$ J. Linacre, ${ }^{144}$ K. Manolopoulos, ${ }^{144}$ D. M. Newbold, ${ }^{144}$ E. Olaiya, ${ }^{144}$ D. Petyt, ${ }^{144}$ T. Reis, ${ }^{144}$ T. Schuh, ${ }^{144}$ C. H. Shepherd-Themistocleous, ${ }^{144}$ I. R. Tomalin, ${ }^{144}$ T. Williams, ${ }^{144}$ R. Bainbridge, ${ }^{145}$ P. Bloch, ${ }^{145}$ S. Bonomally, ${ }^{145}$ J. Borg, ${ }^{145}$ S. Breeze ${ }^{145}$ O. Buchmuller, ${ }^{145}$ V. Cepaitis, ${ }^{145}$ G. S. Chahal, ${ }^{145, \text { hhhh }}$ D. Colling, ${ }^{145}$ P. Dauncey, ${ }^{145}$ G. Davies, ${ }^{145}$ M. Della Negra ${ }^{145}$ S. Fayer, ${ }^{145}$ G. Fedi, ${ }^{145}$ G. Hall, ${ }^{145}$ M. H. Hassanshahi, ${ }^{145}$ G. Iles, ${ }^{145}$ J. Langford, ${ }^{145}$ L. Lyons, ${ }^{145}$ A.-M. Magnan, ${ }^{145}$ S. Malik, ${ }^{145}$ A. Martelli, ${ }^{145}$ D. G. Monk, ${ }^{145}$ J. Nash, ${ }^{145, \text { iiii }}$ M. Pesaresi, ${ }^{145}$ D. M. Raymond, ${ }^{145}$ A. Richards, ${ }^{145}$ A. Rose, ${ }^{145}$ E. Scott, ${ }^{145}$ C. Seez, ${ }^{145}$ A. Shtipliyski, ${ }^{145}$

A. Tapper ${ }^{145}$ K. Uchida ${ }^{145}$ T. Virdee,${ }^{145, u}$ M. Vojinovic, ${ }^{145}$ N. Wardle, ${ }^{145}$ S. N. Webb, ${ }^{145}$ D. Winterbottom, ${ }^{145}$

K. Coldham, ${ }^{146}$ J. E. Cole ${ }^{146}$ A. Khan, ${ }^{146}$ P. Kyberd, ${ }^{146}$ I. D. Reid ${ }^{146}$ L. Teodorescu, ${ }^{146}$ S. Zahid, ${ }^{146}$ S. Abdullin, ${ }^{147}$ A. Brinkerhoff, ${ }^{147}$ B. Caraway, ${ }^{147}$ J. Dittmann, ${ }^{147}$ K. Hatakeyama, ${ }^{147}$ A. R. Kanuganti, ${ }^{147}$ B. McMaster, ${ }^{147}$ N. Pastika, ${ }^{147}$ M. Saunders, ${ }^{147}$ S. Sawant, ${ }^{147}$ C. Sutantawibul, ${ }^{147}$ J. Wilson, ${ }^{147}$ R. Bartek, ${ }^{148}$ A. Dominguez, ${ }^{148}$ R. Uniyal, ${ }^{148}$ A. M. Vargas Hernandez, ${ }^{148}$ A. Buccilli, ${ }^{149}$ S. I. Cooper, ${ }^{149}$ D. Di Croce, ${ }^{149}$ S. V. Gleyzer, ${ }^{149}$ C. Henderson, ${ }^{149}$ C. U. Perez, ${ }^{149}$ P. Rumerio, ${ }^{149, j \mathrm{jjj}}$ C. West, ${ }^{149}$ A. Akpinar, ${ }^{150}$ A. Albert, ${ }^{150}$ D. Arcaro, ${ }^{150}$ C. Cosby, ${ }^{150}$ Z. Demiragli, ${ }^{150}$ E. Fontanesi, ${ }^{150}$ D. Gastler, ${ }^{150}$ S. May, ${ }^{150}$ J. Rohlf, ${ }^{150}$ K. Salyer, ${ }^{150}$ D. Sperka, ${ }^{150}$ D. Spitzbart, ${ }^{150}$ I. Suarez, ${ }^{150}$ A. Tsatsos, ${ }^{150}$ S. Yuan, ${ }^{150}$ 
D. Zou, ${ }^{150}$ G. Benelli, ${ }^{151}$ B. Burkle,${ }^{151}$ X. Coubez,${ }^{151, v}$ D. Cutts, ${ }^{151}$ M. Hadley, ${ }^{151}$ U. Heintz, ${ }^{151}$ J. M. Hogan, ${ }^{151, k k k k}$ G. Landsberg, ${ }^{151}$ K. T. Lau, ${ }^{151}$ M. Lukasik, ${ }^{151}$ J. Luo, ${ }^{151}$ M. Narain, ${ }^{151}$ S. Sagir, ${ }^{151,1111}$ E. Usai, ${ }^{151}$ W. Y. Wong, ${ }^{151}$ X. Yan, ${ }^{151}$ D. Yu, ${ }^{151}$ W. Zhang, ${ }^{151}$ J. Bonilla, ${ }^{152}$ C. Brainerd, ${ }^{152}$ R. Breedon, ${ }^{152}$ M. Calderon De La Barca Sanchez, ${ }^{152}$ M. Chertok, ${ }^{152}$ J. Conway, ${ }^{152}$ P. T. Cox, ${ }^{152}$ R. Erbacher, ${ }^{152}$ G. Haza, ${ }^{152}$ F. Jensen, ${ }^{152}$ O. Kukral,${ }^{152}$ R. Lander, ${ }^{152}$ M. Mulhearn, ${ }^{152}$ D. Pellett, ${ }^{152}$ B. Regnery, ${ }^{152}$ D. Taylor, ${ }^{152}$ Y. Yao ${ }^{152}$ F. Zhang, ${ }^{152}$ M. Bachtis,${ }^{153}$ R. Cousins, ${ }^{153}$ A. Datta, ${ }^{153}$ D. Hamilton,${ }^{153}$ J. Hauser, ${ }^{153}$ M. Ignatenko, ${ }^{153}$ M. A. Iqbal, ${ }^{153}$ T. Lam, ${ }^{153}$ W. A. Nash,${ }^{153}$ S. Regnard, ${ }^{153}$ D. Saltzberg, ${ }^{153}$ B. Stone, ${ }^{153}$ V. Valuev ${ }^{153}$ K. Burt, ${ }^{154}$ Y. Chen,${ }^{154}$ R. Clare,${ }^{154}$ J. W. Gary, ${ }^{154}$ M. Gordon, ${ }^{154}$ G. Hanson, ${ }^{154}$ G. Karapostoli, ${ }^{154}$ O. R. Long, ${ }^{154}$ N. Manganelli, ${ }^{154}$ M. Olmedo Negrete, ${ }^{154}$ W. Si,${ }^{154}$ S. Wimpenny, ${ }^{154}$ Y. Zhang, ${ }^{154}$ J. G. Branson, ${ }^{155}$ P. Chang, ${ }^{155}$ S. Cittolin, ${ }^{155}$ S. Cooperstein, ${ }^{155}$ N. Deelen, ${ }^{155}$ D. Diaz, ${ }^{155}$ J. Duarte, ${ }^{155}$ R. Gerosa ${ }^{155}$ L. Giannini, ${ }^{155}$ D. Gilbert, ${ }^{155}$ J. Guiang, ${ }^{155}$ R. Kansal, ${ }^{155}$ V. Krutelyov, ${ }^{155}$ R. Lee, ${ }^{155}$ J. Letts, ${ }^{155}$ M. Masciovecchio, ${ }^{155}$ M. Pieri, ${ }^{155}$ B. V. Sathia Narayanan, ${ }^{155}$ V. Sharma,${ }^{155}$ M. Tadel, ${ }^{155}$ A. Vartak, ${ }^{155}$ F. Würthwein, ${ }^{155}$ Y. Xiang, ${ }^{155}$ A. Yagil, ${ }^{155}$ N. Amin,${ }^{156}$ C. Campagnari, ${ }^{156}$ M. Citron, ${ }^{156}$ A. Dorsett, ${ }^{156}$ V. Dutta, ${ }^{156}$ J. Incandela,${ }^{156}$ M. Kilpatrick, ${ }^{156}$ J. Kim, ${ }^{156}$ B. Marsh, ${ }^{156}$ H. Mei, ${ }^{156}$ M. Oshiro, ${ }^{156}$ M. Quinnan, ${ }^{156}$ J. Richman, ${ }^{156}$ U. Sarica, ${ }^{156}$ F. Setti, ${ }^{156}$ J. Sheplock, ${ }^{156}$ D. Stuart, ${ }^{156}$ S. Wang, ${ }^{156}$ A. Bornheim ${ }^{157}$ O. Cerri, ${ }^{157}$ I. Dutta, ${ }^{157}$ J. M. Lawhorn, ${ }^{157}$ N. Lu, ${ }^{157}$ J. Mao,${ }^{157}$ H. B. Newman, ${ }^{157}$ T. Q. Nguyen, ${ }^{157}$ M. Spiropulu, ${ }^{157}$ N. Suri Jr., ${ }^{157}$ J. R. Vlimant, ${ }^{157}$ C. Wang, ${ }^{157}$ S. Xie, ${ }^{157}$ Z. Zhang, ${ }^{157}$ R. Y. Zhu, ${ }^{157}$ J. Alison, ${ }^{158}$ S. An, ${ }^{158}$ M. B. Andrews, ${ }^{158}$ P. Bryant, ${ }^{158}$ T. Ferguson, ${ }^{158}$ A. Harilal, ${ }^{158}$ C. Liu, ${ }^{158}$ T. Mudholkar, ${ }^{158}$ M. Paulini, ${ }^{158}$ A. Sanchez, ${ }^{158}$ W. Terrill, ${ }^{158}$ J. P. Cumalat, ${ }^{159}$ W. T. Ford, ${ }^{159}$ A. Hassani, ${ }^{159}$ E. MacDonald, ${ }^{159}$ R. Patel, ${ }^{159}$ A. Perloff, ${ }^{159}$ C. Savard, ${ }^{159}$ K. Stenson, ${ }^{159}$ K. A. Ulmer, ${ }^{159}$ S. R. Wagner, ${ }^{159}$ J. Alexander, ${ }^{160}$ S. Bright-thonney, ${ }^{160}$ Y. Cheng, ${ }^{160}$ D. J. Cranshaw, ${ }^{160}$ S. Hogan, ${ }^{160}$ J. Monroy, ${ }^{160}$ J. R. Patterson, ${ }^{160}$ D. Quach, ${ }^{160}$ J. Reichert, ${ }^{160}$ M. Reid, ${ }^{160}$ A. Ryd, ${ }^{160}$ W. Sun, ${ }^{160}$ J. Thom, ${ }^{160}$ P. Wittich, ${ }^{160}$ R. Zou, ${ }^{160}$ M. Albrow, ${ }^{161}$ M. Alyari, ${ }^{161}$ G. Apollinari, ${ }^{161}$ A. Apresyan, ${ }^{161}$ A. Apyan, ${ }^{161}$ S. Banerjee, ${ }^{161}$ L. A. T. Bauerdick, ${ }^{161}$ D. Berry, ${ }^{161}$ J. Berryhill, ${ }^{161}$ P. C. Bhat, ${ }^{161}$ K. Burkett, ${ }^{161}$ J. N. Butler, ${ }^{161}$ A. Canepa,${ }^{161}$ G. B. Cerati, ${ }^{161}$ H. W. K. Cheung, ${ }^{161}$ F. Chlebana, ${ }^{161}$ M. Cremonesi, ${ }^{161}$ K. F. Di Petrillo, ${ }^{161}$ V. D. Elvira, ${ }^{161}$ Y. Feng, ${ }^{161}$ J. Freeman, ${ }^{161}$ Z. Gecse, ${ }^{161}$ L. Gray, ${ }^{161}$ D. Green, ${ }^{161}$ S. Grünendahl, ${ }^{161}$ O. Gutsche, ${ }^{161}$ R. M. Harris, ${ }^{161}$ R. Heller, ${ }^{161}$ T. C. Herwig, ${ }^{161}$ J. Hirschauer, ${ }^{161}$ B. Jayatilaka, ${ }^{161}$ S. Jindariani, ${ }^{161}$ M. Johnson, ${ }^{161}$ U. Joshi, ${ }^{161}$ T. Klijnsma, ${ }^{161}$ B. Klima, ${ }^{161}$ K. H. M. Kwok, ${ }^{161}$ S. Lammel, ${ }^{161}$ D. Lincoln, ${ }^{161}$ R. Lipton, ${ }^{161}$ T. Liu, ${ }^{161}$ C. Madrid ${ }^{161}$ K. Maeshima, ${ }^{161}$ C. Mantilla, ${ }^{161}$ D. Mason, ${ }^{161}$ P. McBride, ${ }^{161}$ P. Merkel, ${ }^{161}$ S. Mrenna, ${ }^{161}$ S. Nahn, ${ }^{161}$ J. Ngadiuba, ${ }^{161}$ V. O’ Dell, ${ }^{161}$ V. Papadimitriou, ${ }^{161}$ K. Pedro, ${ }^{161}$ C. Pena, ${ }^{161, f f f}$ O. Prokofyev,${ }^{161}$ F. Ravera, ${ }^{161}$ A. Reinsvold Hall, ${ }^{161}$ L. Ristori, ${ }^{161}$ E. Sexton-Kennedy, ${ }^{161}$ N. Smith, ${ }^{161}$ A. Soha, ${ }^{161}$ W. J. Spalding, ${ }^{161}$ L. Spiegel, ${ }^{161}$ S. Stoynev, ${ }^{161}$ J. Strait,${ }^{161}$ L. Taylor ${ }^{161}$ S. Tkaczyk, ${ }^{161}$ N. V. Tran, ${ }^{161}$ L. Uplegger, ${ }^{161}$ E. W. Vaandering, ${ }^{161}$ H. A. Weber, ${ }^{161}$ D. Acosta, ${ }^{162}$ P. Avery, ${ }^{162}$ D. Bourilkov, ${ }^{162}$ L. Cadamuro, ${ }^{162}$ V. Cherepanov, ${ }^{162}$ F. Errico, ${ }^{162}$ R. D. Field, ${ }^{162}$ D. Guerrero, ${ }^{162}$ B. M. Joshi, ${ }^{162}$ M. Kim, ${ }^{162}$ E. Koenig, ${ }^{162}$ J. Konigsberg, ${ }^{162}$ A. Korytov, ${ }^{162}$ K. H. Lo, ${ }^{162}$ K. Matchev, ${ }^{162}$ N. Menendez, ${ }^{162}$ G. Mitselmakher, ${ }^{162}$

A. Muthirakalayil Madhu, ${ }^{162}$ N. Rawal, ${ }^{162}$ D. Rosenzweig, ${ }^{162}$ S. Rosenzweig, ${ }^{162}$ J. Rotter, ${ }^{162}$ K. Shi, ${ }^{162}$ J. Sturdy ${ }^{162}$ J. Wang, ${ }^{162}$ E. Yigitbasi, ${ }^{162}$ X. Zuo, ${ }^{162}$ T. Adams, ${ }^{163}$ A. Askew, ${ }^{163}$ R. Habibullah, ${ }^{163}$ V. Hagopian, ${ }^{163}$ K. F. Johnson, ${ }^{163}$ R. Khurana, ${ }^{163}$ T. Kolberg, ${ }^{163}$ G. Martinez, ${ }^{163}$ H. Prosper,${ }^{163}$ C. Schiber, ${ }^{163}$ O. Viazlo, ${ }^{163}$ R. Yohay, ${ }^{163}$ J. Zhang, ${ }^{163}$ M. M. Baarmand, ${ }^{164}$ S. Butalla, ${ }^{164}$ T. Elkafrawy, ${ }^{164, m m m}$ M. Hohlmann, ${ }^{164}$ R. Kumar Verma, ${ }^{164}$ D. Noonan, ${ }^{164}$ M. Rahmani, ${ }^{164}$ F. Yumiceva, ${ }^{164}$ M. R. Adams, ${ }^{165}$ H. Becerril Gonzalez, ${ }^{165}$ R. Cavanaugh, ${ }^{165}$ X. Chen, ${ }^{165}$ S. Dittmer, ${ }^{165}$ O. Evdokimov, ${ }^{165}$ C. E. Gerber, ${ }^{165}$ D. A. Hangal, ${ }^{165}$ D. J. Hofman, ${ }^{165}$ A. H. Merrit, ${ }^{165}$ C. Mills, ${ }^{165}$ G. Oh, ${ }^{165}$ T. Roy, ${ }^{165}$ S. Rudrabhatla, ${ }^{165}$ M. B. Tonjes, ${ }^{165}$ N. Varelas, ${ }^{165}$ J. Viinikainen, ${ }^{165}$ X. Wang, ${ }^{165}$ Z. Wu, ${ }^{165}$ Z. Ye,${ }^{165}$ M. Alhusseini, ${ }^{166}$

K. Dilsiz, ${ }^{166, n n n n}$ R. P. Gandrajula, ${ }^{166}$ O. K. Köseyan, ${ }^{166}$ J.-P. Merlo, ${ }^{166}$ A. Mestvirishvili, ${ }^{166,0000}$ J. Nachtman, ${ }^{166}$ H. Ogul, ${ }^{166, \text { pppp }}$ Y. Onel, ${ }^{166}$ A. Penzo, ${ }^{166}$ C. Snyder, ${ }^{166}$ E. Tiras, ${ }^{166, q q q q}$ O. Amram, ${ }^{167}$ B. Blumenfeld, ${ }^{167}$ L. Corcodilos, ${ }^{167}$ J. Davis, ${ }^{167}$ M. Eminizer, ${ }^{167}$ A. V. Gritsan, ${ }^{167}$ S. Kyriacou ${ }^{167}$ P. Maksimovic, ${ }^{167}$ J. Roskes,${ }^{167}$ M. Swartz, ${ }^{167}$ T. Á. Vámi, ${ }^{167}$ A. Abreu, ${ }^{168}$ J. Anguiano, ${ }^{168}$ C. Baldenegro Barrera, ${ }^{168}$ P. Baringer, ${ }^{168}$ A. Bean, ${ }^{168}$ A. Bylinkin, ${ }^{168}$ Z. Flowers, ${ }^{168}$ T. Isidori, ${ }^{168}$ S. Khalil, ${ }^{168}$ J. King, ${ }^{168}$ G. Krintiras, ${ }^{168}$ A. Kropivnitskaya, ${ }^{168}$ M. Lazarovits, ${ }^{168}$ C. Lindsey, ${ }^{168}$ J. Marquez, ${ }^{168}$ N. Minafra, ${ }^{168}$ M. Murray ${ }^{168}$ M. Nickel, ${ }^{168}$ C. Rogan, ${ }^{168}$ C. Royon, ${ }^{168}$ R. Salvatico, ${ }^{168}$ S. Sanders, ${ }^{168}$ E. Schmitz,${ }^{168}$ C. Smith, ${ }^{168}$ J. D. Tapia Takaki, ${ }^{168}$ Q. Wang, ${ }^{168}$ Z. Warner, ${ }^{168}$ J. Williams, ${ }^{168}$ G. Wilson, ${ }^{168}$ S. Duric, ${ }^{169}$ A. Ivanov, ${ }^{169}$ K. Kaadze, ${ }^{169}$ D. Kim, ${ }^{169}$ Y. Maravin, ${ }^{169}$ T. Mitchell, ${ }^{169}$ A. Modak, ${ }^{169}$ K. Nam,${ }^{169}$ F. Rebassoo, ${ }^{170}$ D. Wright,${ }^{170}$ E. Adams, ${ }^{171}$ A. Baden, ${ }^{171}$ O. Baron, ${ }^{171}$ A. Belloni, ${ }^{171}$ S. C. Eno, ${ }^{171}$ N. J. Hadley, ${ }^{171}$ S. Jabeen, ${ }^{171}$ R. G. Kellogg, ${ }^{171}$ T. Koeth, ${ }^{171}$ A. C. Mignerey, ${ }^{171}$ S. Nabili, ${ }^{171}$ C. Palmer, ${ }^{171}$ M. Seidel, ${ }^{171}$ A. Skuja, ${ }^{171}$ L. Wang, ${ }^{171}$ K. Wong, ${ }^{171}$ D. Abercrombie, ${ }^{172}$ G. Andreassi, ${ }^{172}$ R. Bi,${ }^{172}$ S. Brandt, ${ }^{172}$ W. Busza, ${ }^{172}$ I. A. Cali, ${ }^{172}$ Y. Chen, ${ }^{172}$ M. D' Alfonso, ${ }^{172}$ J. Eysermans, ${ }^{172}$ C. Freer, ${ }^{172}$ 
G. Gomez Ceballos, ${ }^{172}$ M. Goncharov, ${ }^{172}$ P. Harris,${ }^{172}$ M. Hu,${ }^{172}$ M. Klute, ${ }^{172}$ D. Kovalskyi, ${ }^{172}$ J. Krupa,${ }^{172}$ Y.-J. Lee, ${ }^{172}$ C. Mironov, ${ }^{172}$ C. Paus, ${ }^{172}$ D. Rankin, ${ }^{172}$ C. Roland,${ }^{172}$ G. Roland, ${ }^{172}$ Z. Shi, ${ }^{172}$ G. S. F. Stephans, ${ }^{172}$ J. Wang, ${ }^{172}$ Z. Wang, ${ }^{172}$ B. Wyslouch, ${ }^{172}$ R. M. Chatterjee, ${ }^{173}$ A. Evans, ${ }^{173}$ P. Hansen, ${ }^{173}$ J. Hiltbrand, ${ }^{173}$ Sh. Jain, ${ }^{173}$ M. Krohn, ${ }^{173}$ Y. Kubota, ${ }^{173}$ J. Mans, ${ }^{173}$ M. Revering, ${ }^{173}$ R. Rusack, ${ }^{173}$ R. Saradhy, ${ }^{173}$ N. Schroeder,${ }^{173}$ N. Strobbe, ${ }^{173}$ M. A. Wadud, ${ }^{173}$ K. Bloom, ${ }^{174}$ M. Bryson, ${ }^{174}$ S. Chauhan, ${ }^{174}$ D. R. Claes, ${ }^{174}$ C. Fangmeier, ${ }^{174}$ L. Finco, ${ }^{174}$ F. Golf, ${ }^{174}$ C. Joo, ${ }^{174}$ I. Kravchenko, ${ }^{174}$ M. Musich,${ }^{174}$ I. Reed, ${ }^{174}$ J. E. Siado, ${ }^{174}$ G. R. Snow, ${ }^{174, a}$ W. Tabb,${ }^{174}$ F. Yan, ${ }^{174}$ A. G. Zecchinelli, ${ }^{174}$ G. Agarwal, ${ }^{175}$

H. Bandyopadhyay, ${ }^{175}$ L. Hay, ${ }^{175}$ I. Iashvili, ${ }^{175}$ A. Kharchilava, ${ }^{175}$ C. McLean, ${ }^{175}$ D. Nguyen, ${ }^{175}$ J. Pekkanen, ${ }^{175}$ S. Rappoccio, ${ }^{175}$ A. Williams, ${ }^{175}$ G. Alverson, ${ }^{176}$ E. Barberis, ${ }^{176}$ Y. Haddad, ${ }^{176}$ A. Hortiangtham, ${ }^{176}$ J. Li, ${ }^{176}$ G. Madigan, ${ }^{176}$ B. Marzocchi ${ }^{176}$ D. M. Morse,${ }^{176}$ V. Nguyen, ${ }^{176}$ T. Orimoto, ${ }^{176}$ A. Parker,${ }^{176}$ L. Skinnari, ${ }^{176}$ A. Tishelman-Charny, ${ }^{176}$ T. Wamorkar, ${ }^{176}$ B. Wang, ${ }^{176}$ A. Wisecarver, ${ }^{176}$ D. Wood, ${ }^{176}$ S. Bhattacharya, ${ }^{177}$ J. Bueghly, ${ }^{177}$ Z. Chen, ${ }^{177}$ A. Gilbert, ${ }^{177}$ T. Gunter, ${ }^{177}$ K. A. Hahn, ${ }^{177}$ Y. Liu, ${ }^{177}$ N. Odell, ${ }^{177}$ M. H. Schmitt, ${ }^{177}$ M. Velasco, ${ }^{177}$ R. Band,${ }^{178}$ R. Bucci, ${ }^{178}$ A. Das, ${ }^{178}$ N. Dev, ${ }^{178}$ R. Goldouzian, ${ }^{178}$ M. Hildreth, ${ }^{178}$ K. Hurtado Anampa, ${ }^{178}$ C. Jessop, ${ }^{178}$ K. Lannon, ${ }^{178}$ J. Lawrence, ${ }^{178}$ N. Loukas, ${ }^{178}$ D. Lutton, ${ }^{178}$ N. Marinelli, ${ }^{178}$ I. Mcalister, ${ }^{178}$ T. McCauley, ${ }^{178}$ C. Mcgrady, ${ }^{178}$ K. Mohrman, ${ }^{178}$ Y. Musienko, ${ }^{178, y y}$ R. Ruchti, ${ }^{178}$ P. Siddireddy, ${ }^{178}$ A. Townsend, ${ }^{178}$ M. Wayne, ${ }^{178}$ A. Wightman,${ }^{178}$ M. Zarucki, ${ }^{178}$ L. Zygala, ${ }^{178}$ B. Bylsma, ${ }^{179}$ B. Cardwell, ${ }^{179}$ L. S. Durkin, ${ }^{179}$ B. Francis,${ }^{179}$ C. Hill, ${ }^{179}$ M. Nunez Ornelas, ${ }^{179}$ K. Wei, ${ }^{179}$ B. L. Winer, ${ }^{179}$ B. R. Yates, ${ }^{179}$ F. M. Addesa, ${ }^{180}$ B. Bonham, ${ }^{180}$ P. Das, ${ }^{180}$ G. Dezoort, ${ }^{180}$ P. Elmer,${ }^{180}$ A. Frankenthal, ${ }^{180}$ B. Greenberg, ${ }^{180}$ N. Haubrich, ${ }^{180}$ S. Higginbotham, ${ }^{180}$ A. Kalogeropoulos,${ }^{180}$ G. Kopp ${ }^{180}$ S. Kwan, ${ }^{180}$ D. Lange, ${ }^{180}$ D. Marlow, ${ }^{180}$ K. Mei, ${ }^{180}$ I. Ojalvo, ${ }^{180}$ J. Olsen, ${ }^{180}$ D. Stickland, ${ }^{180}$ C. Tully, ${ }^{180}$ S. Malik, ${ }^{181}$ S. Norberg, ${ }^{181}$ A. S. Bakshi, ${ }^{182}$ V. E. Barnes, ${ }^{182}$ R. Chawla, ${ }^{182}$ S. Das, ${ }^{182}$ L. Gutay, ${ }^{182}$ M. Jones, ${ }^{182}$ A. W. Jung, ${ }^{182}$ S. Karmarkar, ${ }^{182}$ D. Kondratyev, ${ }^{182}$ M. Liu, ${ }^{182}$ G. Negro, ${ }^{182}$ N. Neumeister, ${ }^{182}$ G. Paspalaki, ${ }^{182}$ C. C. Peng, ${ }^{182}$ S. Piperov, ${ }^{182}$ A. Purohit, ${ }^{182}$ J. F. Schulte, ${ }^{182}$ M. Stojanovic, ${ }^{182, \mathrm{r}}$ J. Thieman, ${ }^{182}$ F. Wang, ${ }^{182}$ R. Xiao, ${ }^{182}$ W. Xie, ${ }^{182}$ J. Dolen, ${ }^{183}$ N. Parashar, ${ }^{183}$ A. Baty, ${ }^{184}$ M. Decaro, ${ }^{184}$ S. Dildick, ${ }^{184}$ K. M. Ecklund, ${ }^{184}$ S. Freed, ${ }^{184}$ P. Gardner, ${ }^{184}$ F. J. M. Geurts, ${ }^{184}$ A. Kumar, ${ }^{184}$ W. Li, ${ }^{184}$ B. P. Padley, ${ }^{184}$ R. Redjimi, ${ }^{184}$ W. Shi, ${ }^{184}$ A. G. Stahl Leiton, ${ }^{184}$ S. Yang, ${ }^{184}$ L. Zhang, ${ }^{184}$ Y. Zhang, ${ }^{184}$ A. Bodek ${ }^{185}$ P. de Barbaro, ${ }^{185}$ R. Demina, ${ }^{185}$ J. L. Dulemba, ${ }^{185}$ C. Fallon, ${ }^{185}$ T. Ferbel, ${ }^{185}$ M. Galanti, ${ }^{185}$ A. Garcia-Bellido, ${ }^{185}$ O. Hindrichs, ${ }^{185}$ A. Khukhunaishvili, ${ }^{185}$ E. Ranken, ${ }^{185}$ R. Taus, ${ }^{185}$ B. Chiarito, ${ }^{186}$ J. P. Chou, ${ }^{186}$ A. Gandrakota, ${ }^{186}$ Y. Gershtein, ${ }^{186}$ E. Halkiadakis, ${ }^{186}$ A. Hart, ${ }^{186}$ M. Heindl, ${ }^{186}$ O. Karacheban ${ }^{186, y}$ I. Laflotte, ${ }^{186}$ A. Lath, ${ }^{186}$ R. Montalvo, ${ }^{186}$ K. Nash, ${ }^{186}$ M. Osherson, ${ }^{186}$ S. Salur, ${ }^{186}$ S. Schnetzer, ${ }^{186}$ S. Somalwar, ${ }^{186}$ R. Stone, ${ }^{186}$ S. A. Thayil, ${ }^{186}$ S. Thomas, ${ }^{186}$ H. Wang, ${ }^{186}$ H. Acharya, ${ }^{187}$ A. G. Delannoy, ${ }^{187}$ S. Fiorendi, ${ }^{187}$ S. Spanier, ${ }^{187}$ O. Bouhali, ${ }^{188, \text { rrrr }}$ M. Dalchenko, ${ }^{188}$ A. Delgado, ${ }^{188}$

R. Eusebi, ${ }^{188}$ J. Gilmore, ${ }^{188}$ T. Huang, ${ }^{188}$ T. Kamon, ${ }^{188, \text { sss }}$ H. Kim,${ }^{188}$ S. Luo,${ }^{188}$ S. Malhotra, ${ }^{188}$ R. Mueller, ${ }^{188}$

D. Overton, ${ }^{188}$ D. Rathjens, ${ }^{188}$ A. Safonov ${ }^{188}$ N. Akchurin, ${ }^{189}$ J. Damgov, ${ }^{189}$ V. Hegde, ${ }^{189}$ S. Kunori, ${ }^{189}$ K. Lamichhane,${ }^{189}$ S. W. Lee, ${ }^{189}$ T. Mengke, ${ }^{189}$ S. Muthumuni, ${ }^{189}$ T. Peltola, ${ }^{189}$ I. Volobouev, ${ }^{189}$ Z. Wang, ${ }^{189}$ A. Whitbeck, ${ }^{189}$ E. Appelt, ${ }^{190}$ S. Greene, ${ }^{190}$ A. Gurrola,${ }^{190}$ W. Johns,${ }^{190}$ A. Melo, ${ }^{190}$ H. Ni ${ }^{190}$ K. Padeken, ${ }^{190}$ F. Romeo, ${ }^{190}$ P. Sheldon,${ }^{190}$ S. Tuo, ${ }^{190}$ J. Velkovska, ${ }^{190}$ M. W. Arenton, ${ }^{191}$ B. Cox,${ }^{191}$ G. Cummings, ${ }^{191}$ J. Hakala, ${ }^{191}$ R. Hirosky, ${ }^{191}$ M. Joyce, ${ }^{191}$ A. Ledovskoy, ${ }^{191}$ A. Li, ${ }^{191}$ C. Neu, ${ }^{191}$ B. Tannenwald, ${ }^{191}$ S. White, ${ }^{191}$ E. Wolfe, ${ }^{191}$ N. Poudyal,${ }^{192}$ K. Black, ${ }^{193}$ T. Bose,${ }^{193}$ C. Caillol,,${ }^{193}$ S. Dasu, ${ }^{193}$ I. De Bruyn, ${ }^{193}$ P. Everaerts, ${ }^{193}$ F. Fienga, ${ }^{193}$ C. Galloni, ${ }^{193}$ H. He,${ }^{193}$ M. Herndon, ${ }^{193}$ A. Hervé,${ }^{193}$ U. Hussain, ${ }^{193}$ A. Lanaro, ${ }^{193}$ A. Loeliger, ${ }^{193}$ R. Loveless, ${ }^{193}$ J. Madhusudanan Sreekala, ${ }^{193}$ A. Mallampalli, ${ }^{193}$ A. Mohammadi, ${ }^{193}$ D. Pinna, ${ }^{193}$ A. Savin, ${ }^{193}$ V. Shang,,${ }^{193}$ V. Sharma, ${ }^{193}$ W. H. Smith, ${ }^{193}$ D. Teague, ${ }^{193}$

S. Trembath-Reichert, ${ }^{193}$ and W. Vetens ${ }^{193}$

(CMS Collaboration)

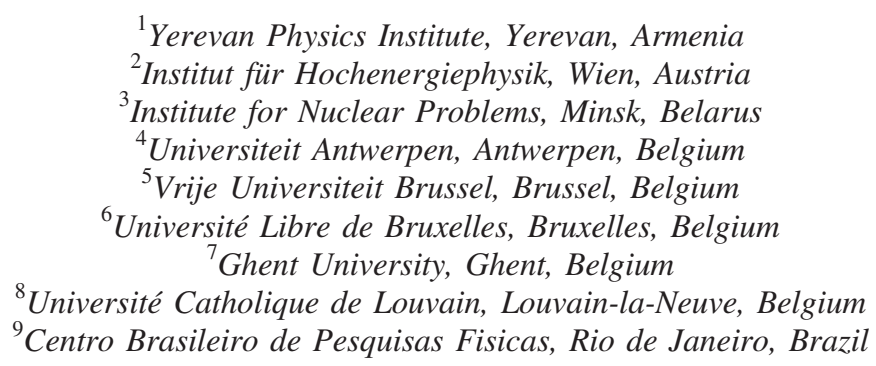




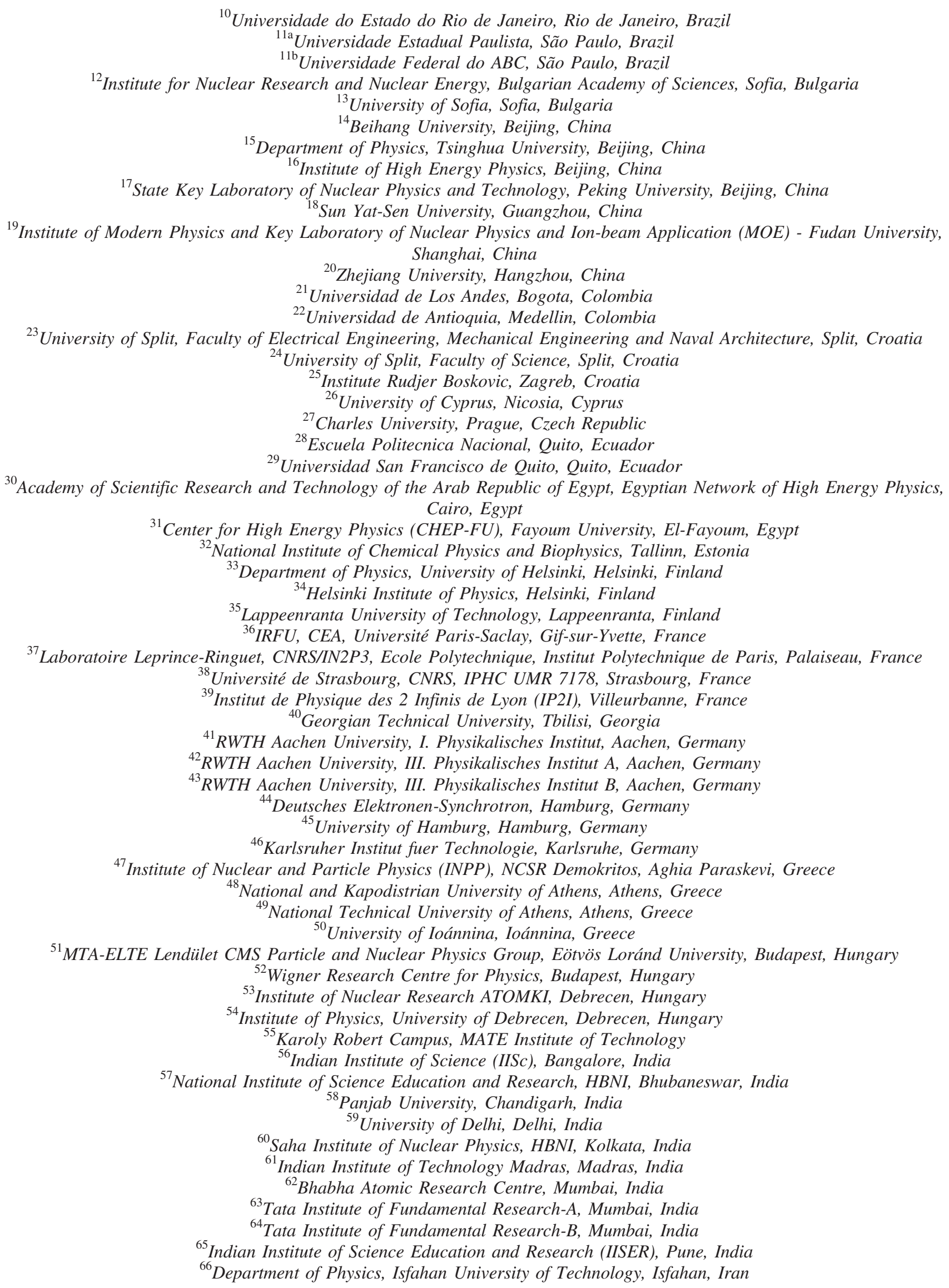


${ }^{67}$ Institute for Research in Fundamental Sciences (IPM), Tehran, Iran

${ }^{68}$ University College Dublin, Dublin, Ireland

${ }^{69 \mathrm{a}}$ INFN Sezione di Bari, Bari, Italy

${ }^{69 \mathrm{~b}}$ Università di Bari, Bari, Italy

${ }^{69 \mathrm{c}}$ Politecnico di Bari, Bari, Italy

${ }^{70 a}$ INFN Sezione di Bologna, Bologna, Italy

${ }^{70 \mathrm{~b}}$ Università di Bologna, Bologna, Italy

${ }^{71 a}$ INFN Sezione di Catania, Catania, Italy

${ }^{71 b}$ Università di Catania, Catania, Italy

${ }^{72 \mathrm{a}}$ INFN Sezione di Firenze, Firenze, Italy

${ }^{72 \mathrm{~b}}$ Università di Firenze, Firenze, Italy

${ }^{73}$ INFN Laboratori Nazionali di Frascati, Frascati, Italy

${ }^{74 a}$ INFN Sezione di Genova, Genova, Italy

${ }^{74 \mathrm{~b}}$ Università di Genova, Genova, Italy

${ }^{75 a}$ INFN Sezione di Milano-Bicocca, Milano, Italy

${ }^{75 b}$ Università di Milano-Bicocca, Milano, Italy

${ }^{76 a}$ INFN Sezione di Napoli, Napoli, Italy

${ }^{76 \mathrm{~b}}$ Università di Napoli 'Federico II', Napoli, Italy

${ }^{76 c}$ Università della Basilicata, Potenza, Italy

${ }^{76 \mathrm{~d}}$ Università G. Marconi, Roma, Italy

${ }^{77 a}$ INFN Sezione di Padova, Padova, Italy

${ }^{77 b}$ Università di Padova, Padova, Italy

${ }^{77 \mathrm{c}}$ Università di Trento, Trento, Italy

${ }^{78 a}$ INFN Sezione di Pavia

${ }^{78 \mathrm{~b}}$ Università di Pavia

${ }^{79 a}$ INFN Sezione di Perugia, Perugia, Italy

${ }^{79 \mathrm{~b}}$ Università di Perugia, Perugia, Italy

${ }^{80 \mathrm{a}}$ INFN Sezione di Pisa, Pisa, Italy

${ }^{80 \mathrm{~b}}$ Università di Pisa, Pisa, Italy

${ }^{80 \mathrm{c}}$ Scuola Normale Superiore di Pisa, Pisa, Italy

${ }^{80 \mathrm{~d}}$ Università di Siena, Siena, Italy

${ }^{81 a}$ INFN Sezione di Roma, Rome, Italy

${ }^{81 \mathrm{~b}}$ Sapienza Università di Roma, Rome, Italy

${ }^{82 \mathrm{a}}$ INFN Sezione di Torino, Torino, Italy

${ }^{82 \mathrm{~b}}$ Università di Torino, Torino, Italy

${ }^{82 \mathrm{c}}$ Università del Piemonte Orientale, Novara, Italy

${ }^{83 a}$ INFN Sezione di Trieste, Trieste, Italy

${ }^{83 \mathrm{~b}}$ Università di Trieste, Trieste, Italy

${ }^{84}$ Kyungpook National University, Daegu, Korea

${ }^{85}$ Chonnam National University, Institute for Universe and Elementary Particles, Kwangju, Korea

${ }^{86}$ Hanyang University, Seoul, Korea

${ }^{87}$ Korea University, Seoul, Korea

${ }^{88}$ Kyung Hee University, Department of Physics, Seoul, Republic of Korea

${ }^{89}$ Sejong University, Seoul, Korea

${ }^{90}$ Seoul National University, Seoul, Korea

${ }^{91}$ University of Seoul, Seoul, Korea

${ }^{92}$ Yonsei University, Department of Physics, Seoul, Korea

${ }^{93}$ Sungkyunkwan University, Suwon, Korea

${ }^{94}$ College of Engineering and Technology, American University of the Middle East (AUM), Egaila, Kuwait

${ }^{95}$ Riga Technical University, Riga, Latvia

${ }^{96}$ Vilnius University, Vilnius, Lithuania

${ }^{97}$ National Centre for Particle Physics, Universiti Malaya, Kuala Lumpur, Malaysia

${ }^{98}$ Universidad de Sonora (UNISON), Hermosillo, Mexico

${ }^{99}$ Centro de Investigacion y de Estudios Avanzados del IPN, Mexico City, Mexico

${ }^{100}$ Universidad Iberoamericana, Mexico City, Mexico

${ }^{101}$ Benemerita Universidad Autonoma de Puebla, Puebla, Mexico

${ }^{102}$ University of Montenegro, Podgorica, Montenegro

${ }^{103}$ University of Auckland, Auckland, New Zealand

${ }^{104}$ University of Canterbury, Christchurch, New Zealand

${ }^{105}$ National Centre for Physics, Quaid-I-Azam University, Islamabad, Pakistan 
${ }^{106}$ AGH University of Science and Technology Faculty of Computer Science, Electronics and Telecommunications, Krakow, Poland

${ }^{107}$ National Centre for Nuclear Research, Swierk, Poland

${ }^{108}$ Institute of Experimental Physics, Faculty of Physics, University of Warsaw, Warsaw, Poland

${ }^{109}$ Laboratório de Instrumentação e Física Experimental de Partículas, Lisboa, Portugal

${ }^{110}$ Joint Institute for Nuclear Research, Dubna, Russia

${ }^{111}$ Petersburg Nuclear Physics Institute, Gatchina (St. Petersburg), Russia

${ }^{112}$ Institute for Nuclear Research, Moscow, Russia

${ }^{113}$ Institute for Theoretical and Experimental Physics named by A.I. Alikhanov of NRC 'Kurchatov Institute', Moscow, Russia

${ }^{114}$ Moscow Institute of Physics and Technology, Moscow, Russia

${ }^{115}$ National Research Nuclear University 'Moscow Engineering Physics Institute' (MEPhI), Moscow, Russia

${ }^{116}$ P.N. Lebedev Physical Institute, Moscow, Russia

${ }^{117}$ Skobeltsyn Institute of Nuclear Physics, Lomonosov Moscow State University, Moscow, Russia

${ }^{118}$ Novosibirsk State University (NSU), Novosibirsk, Russia

${ }^{119}$ Institute for High Energy Physics of National Research Centre 'Kurchatov Institute', Protvino, Russia

${ }^{120}$ National Research Tomsk Polytechnic University, Tomsk, Russia

${ }^{121}$ Tomsk State University, Tomsk, Russia

${ }^{122}$ University of Belgrade: Faculty of Physics and VINCA Institute of Nuclear Sciences, Belgrade, Serbia

${ }^{123}$ Centro de Investigaciones Energéticas Medioambientales y Tecnológicas (CIEMAT), Madrid, Spain

${ }^{124}$ Universidad Autónoma de Madrid, Madrid, Spain

${ }^{125}$ Universidad de Oviedo, Instituto Universitario de Ciencias y Tecnologías Espaciales de Asturias (ICTEA), Oviedo, Spain

${ }^{126}$ Instituto de Física de Cantabria (IFCA), CSIC-Universidad de Cantabria, Santander, Spain

${ }^{127}$ University of Colombo, Colombo, Sri Lanka

${ }^{128}$ University of Ruhuna, Department of Physics, Matara, Sri Lanka

${ }^{129}$ CERN, European Organization for Nuclear Research, Geneva, Switzerland

${ }^{130}$ Paul Scherrer Institut, Villigen, Switzerland

${ }^{131}$ ETH Zurich - Institute for Particle Physics and Astrophysics (IPA), Zurich, Switzerland

${ }^{132}$ Universität Zürich, Zurich, Switzerland

${ }^{133}$ National Central University, Chung-Li, Taiwan

${ }^{134}$ National Taiwan University (NTU), Taipei, Taiwan

${ }^{135}$ Chulalongkorn University, Faculty of Science, Department of Physics, Bangkok, Thailand

${ }^{136}$ Çukurova University, Physics Department, Science and Art Faculty, Adana, Turkey

${ }^{137}$ Middle East Technical University, Physics Department, Ankara, Turkey

${ }^{138}$ Bogazici University, Istanbul, Turkey

${ }^{139}$ Istanbul Technical University, Istanbul, Turkey

${ }^{140}$ Istanbul University, Istanbul, Turkey

${ }^{141}$ Institute for Scintillation Materials of National Academy of Science of Ukraine, Kharkov, Ukraine

${ }^{142}$ National Scientific Center, Kharkov Institute of Physics and Technology, Kharkov, Ukraine

${ }^{143}$ University of Bristol, Bristol, United Kingdom

${ }^{144}$ Rutherford Appleton Laboratory, Didcot, United Kingdom

${ }^{145}$ Imperial College, London, United Kingdom

${ }^{146}$ Brunel University, Uxbridge, United Kingdom

${ }^{147}$ Baylor University, Waco, Texas, USA

${ }^{148}$ Catholic University of America, Washington, DC, USA

${ }^{149}$ The University of Alabama, Tuscaloosa, Alabama, USA

${ }^{150}$ Boston University, Boston, Massachusetts, USA

${ }^{151}$ Brown University, Providence, Rhode Island, USA

${ }^{152}$ University of California, Davis, Davis, California, USA

${ }^{153}$ University of California, Los Angeles, California, USA

${ }^{154}$ University of California, Riverside, Riverside, California, USA

${ }^{155}$ University of California, San Diego, La Jolla, California, USA

${ }^{156}$ University of California, Santa Barbara - Department of Physics, Santa Barbara, California, USA

${ }^{157}$ California Institute of Technology, Pasadena, California, USA

${ }^{158}$ Carnegie Mellon University, Pittsburgh, Pennsylvania, USA

${ }^{159}$ University of Colorado Boulder, Boulder, Colorado, USA

${ }^{160}$ Cornell University, Ithaca, New York, USA

${ }^{161}$ Fermi National Accelerator Laboratory, Batavia, Illinois, USA

${ }^{162}$ University of Florida, Gainesville, Florida, USA

${ }^{163}$ Florida State University, Tallahassee, Florida, USA

${ }^{164}$ Florida Institute of Technology, Melbourne, Florida, USA

${ }^{165}$ University of Illinois at Chicago (UIC), Chicago, Illinois, USA 


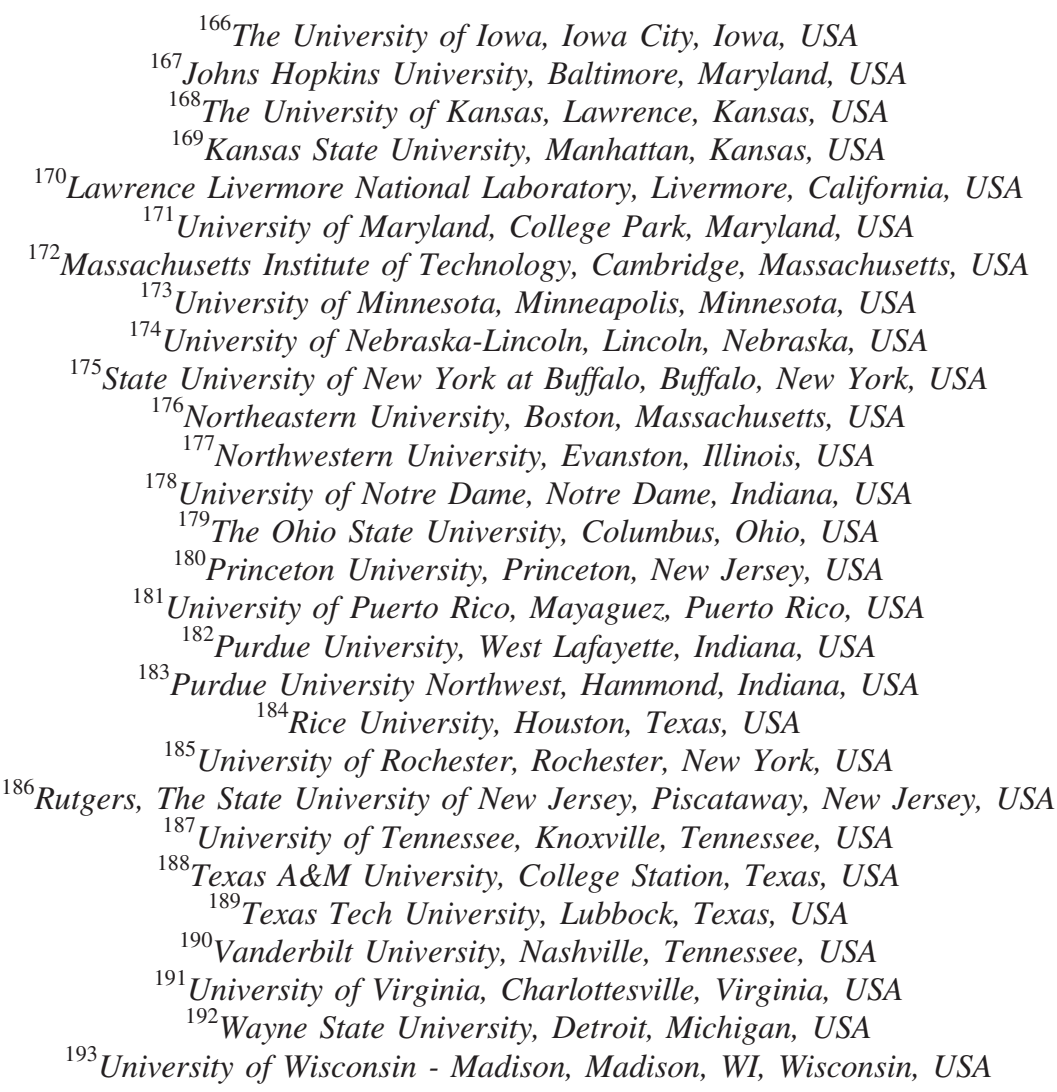

${ }^{\mathrm{a}}$ Deceased.

${ }^{\mathrm{b}}$ Also at TU Wien.

${ }^{c}$ Also at Institute of Basic and Applied Sciences, Faculty of Engineering, Arab Academy for Science, Technology and Maritime Transport, Alexandria, Egypt.

${ }^{\mathrm{d}}$ Also at Université Libre de Bruxelles, Bruxelles, Belgium.

${ }^{\mathrm{e}}$ Also at Universidade Estadual de Campinas, Campinas, Brazil.

${ }^{\mathrm{f}}$ Also at Federal University of Rio Grande do Sul, Porto Alegre, Brazil.

${ }^{\mathrm{g}}$ Also at University of Chinese Academy of Sciences, Bejing, China.

${ }^{\mathrm{h}}$ Also at Department of Physics, Tsinghua University, Beijing, China.

${ }^{1}$ Also at Universidade Federal do Mato Grosso do Sul, Nova Andradina, Mato Grosso do Sul, Brazil.

${ }^{\mathrm{j}}$ Also at The University of Iowa, Iowa City, Iowa, USA.

${ }^{k}$ Also at Nanjing Normal University Department of Physics.

${ }^{1}$ Also at University of Chinese Academy of Sciences, Bejing, China.

${ }^{\mathrm{m}}$ Also at Institute for Theoretical and Experimental Physics named by A.I. Alikhanov of NRC 'Kurchatov Institute', Moscow, Russia.

${ }^{\mathrm{n}}$ Also at Joint Institute for Nuclear Research, Dubna, Russia.

${ }^{\circ}$ Also at Helwan University, Cairo, Egypt.

${ }^{\mathrm{p}}$ Also at Zewail City of Science and Technology, Zewail, Egypt.

${ }^{\mathrm{q}}$ Also at British University in Egypt, Cairo, Egypt.

${ }^{\mathrm{r}}$ Also at Purdue University, West Lafayette, Indiana, USA.

${ }^{\mathrm{s}}$ Also at Université de Haute Alsace, Mulhouse, France.

${ }^{t}$ Also at Erzincan Binali Yildirim University, Erzincan, Turkey.

"Also at CERN, European Organization for Nuclear Research, Geneva, Switzerland.

${ }^{v}$ Also at RWTH Aachen University, III. Physikalisches Institut A, Aachen, Germany.

${ }^{\mathrm{w}}$ Also at University of Hamburg, Hamburg, Germany.

${ }^{x}$ Also at Department of Physics, Isfahan University of Technology, Isfahan, Iran.

${ }^{y}$ Also at Brandenburg University of Technology, Cottbus, Germany.

${ }^{\mathrm{z}}$ Also at Forschungszentrum Jülich, Jülich, Germany.

${ }^{a a}$ Also at Physics Department, Faculty of Science, Assiut University, Assiut, Egypt.

${ }^{\mathrm{bb}}$ Also at Karoly Robert Campus, MATE Institute of Technology, Gyöngyös, Hungary.

${ }^{c c}$ Also at Institute of Physics, University of Debrecen, Debrecen, Hungary. 


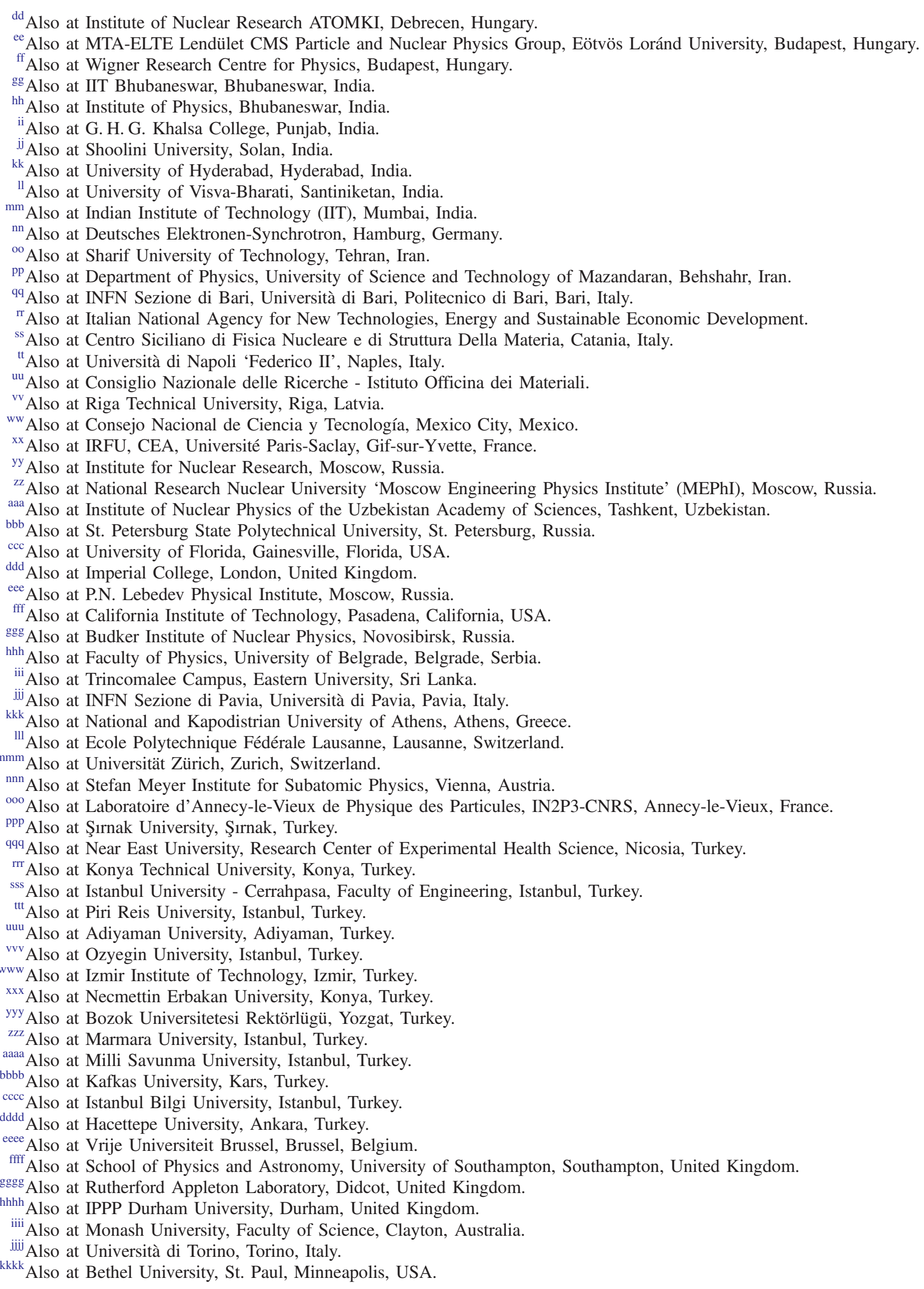




\footnotetext{
${ }^{1111}$ Also at Karamanoğlu Mehmetbey University, Karaman, Turkey.

${ }^{\mathrm{mmmm}}$ Also at Ain Shams University, Cairo, Egypt.

${ }^{\mathrm{nnnn}}$ Also at Bingol University, Bingol, Turkey.

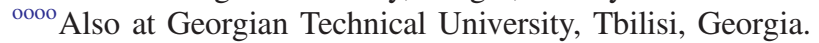

${ }^{\mathrm{pppp}}$ Also at Sinop University, Sinop, Turkey.

${ }^{\mathrm{qqqq}}$ Also at Erciyes University, Kayseri, Turkey.

${ }^{\mathrm{rrrr}}$ Also at Texas A\&M University at Qatar, Doha, Qatar.

${ }^{\text {ssss }}$ Also at Kyungpook National University, Daegu, Korea.
} 